ARTICLE

Received 25 Nov 2014 | Accepted 24 Feb 2015 | Published 13 Apr $2015 \quad$ DOl: 10.1038/ncomms7745

\title{
G-CSF supports long-term muscle regeneration in mouse models of muscular dystrophy
}

Nozomi Hayashiji, Shinsuke Yuasa', Yuko Miyagoe-Suzuki², Mie Hara', Naoki Ito², Hisayuki Hashimoto', Dai Kusumoto', Tomohisa Seki ${ }^{1}$, Shugo Tohyama', Masaki Kodaira', Akira Kunitomi', Shin Kashimura', Makoto Takei ${ }^{1}$, Yuki Saito', Shinichiro Okata1, Toru Egashira', Jin Endo', Toshikuni Sasaoka ${ }^{3}$, Shin'ichi Takeda ${ }^{2} \&$ Keiichi Fukuda ${ }^{1}$

Duchenne muscular dystrophy (DMD) is a chronic and life-threatening disease that is initially supported by muscle regeneration but eventually shows satellite cell exhaustion and muscular dysfunction. The life-long maintenance of skeletal muscle homoeostasis requires the satellite stem cell pool to be preserved. Asymmetric cell division plays a pivotal role in the maintenance of the satellite cell pool. Here we show that granulocyte colony-stimulating factor receptor (G-CSFR) is asymmetrically expressed in activated satellite cells. G-CSF positively affects the satellite cell population during multiple stages of differentiation in ex vivo cultured fibres. G-CSF could be important in developing an effective therapy for DMD based on its potential to modulate the supply of multiple stages of regenerated myocytes. This study shows that the G-CSF-G-CSFR axis is fundamentally important for long-term muscle regeneration, functional maintenance and lifespan extension in mouse models of DMD with varying severities.

\footnotetext{
${ }^{1}$ Department of Cardiology, Keio University School of Medicine, 35-Shinanomachi Shinjuku-ku, Tokyo 160-8582, Japan. ${ }^{2}$ Department of Molecular Therapy, National Institute of Neuroscience, National Center of Neurology and Psychiatry, Tokyo 187-8502, Japan. ${ }^{3}$ Department of Comparative and Experimental Medicine, Brain Research Institute, Niigata University, Niigata 951-8585, Japan. Correspondence and requests for materials should be addressed to S.Y. (email: yuasa@a8.keio.jp).
} 
A dult skeletal muscle has its own stem cell population known as satellite cells (SCs). After muscle injury, quiescent SCs are activated and then proliferate and differentiate into mature skeletal muscle to ensure that muscle function is recovered. SCs are at the top of the hierarchy of differentiating stages in adult myocytes, followed by myoblasts, immature myocytes and matured myocytes. Thus, maintaining the homoeostasis of skeletal muscle function over the long term requires a well-preserved population of SCs. However, SCs are still heterogenic, and among the distinct differentiation stages, the satellite stem cell population must be maintained to preserve their stemness, that is, long-term self-renewal and differentiation abilities. Accumulating data indicate that SCs maintain their population by asymmetric cell division ${ }^{1-4}$. The different differentiation stages of SCs are distinguishable by the expression of specific markers such as PAX7 and MYOD; PAX7 + /MYOD - cells are quiescent, PAX7 + /MYOD + cells are activated and PAX7 - /MYOD + cells are differentiating. Interestingly, some activated SCs exhibit asymmetric MYOD distribution within the cells, and each daughter cell is either $\mathrm{PAX} 7+/ \mathrm{MYOD}+$ or $\mathrm{PAX} 7+/ \mathrm{MYOD}-$, the latter of which retains the quiescent state ${ }^{5}$. Such evidence indicated that asymmetric protein distribution produces asymmetric cell division, which has a critical role in maintaining the SC population. In our previous screen for myocyte differentiationpromoting factors ${ }^{6,7}$, we noted markedly elevated expression of granulocyte colony-stimulating factor receptor (G-CSFR, encoded by $c s f 3 r$ ) in the developing somite ${ }^{8}$. Furthermore, G-CSFR was transiently expressed in regenerating myocytes of adult injured skeletal muscle, and extrinsic G-CSF supported short-term muscle regeneration in cardiotoxin-induced skeletal muscle injury ${ }^{9}$ and crush injury ${ }^{10}$. However, although we found high G-CSFR expression in regenerating immature myocytes, whether G-CSFR would be expressed in SCs and whether G-CSF signalling would affect the SC population in skeletal muscle remain unclear.

Duchenne muscular dystrophy (DMD) is one of the most common inheritable muscle diseases in humans ${ }^{11,12}$. It is an $\mathrm{X}$-linked progressive disease that affects approximately one in 3,500 male live births ${ }^{13}$. DMD is caused by a mutation in the gene encoding dystrophin, which links the internal muscle cytoskeleton to the extracellular matrix, enabling the lateral transmission of force from within muscle cells to the surrounding matrix. Mutations in the dystrophin gene lead to dystrophin deficiency at the myofibre membrane and progressive muscle fibre degeneration ${ }^{14,15}$. The absence of dystrophin increases the fragility of the sarcolemma, which is susceptible to even mild stress $^{16}$. Subsequent injury results in myofibre necrosis, followed by repetitive degeneration and regeneration supported by innate SCs. In DMD, muscle regeneration initially supports the mutation-driven functional impairment; however, such regeneration is eventually attenuated due to stem cell exhaustion ${ }^{17}$. Therapy that induces effective long-term skeletal muscle regeneration is, therefore, crucially needed for DMD.

Long-term muscle regenerative therapy for conditions such as DMD relies on increasing the pool of functional myocytes and maintaining the pool of SCs. We showed that G-CSFR is asymmetrically distributed in activated SCs, and that G-CSF increases the population of SCs via the G-CSF-G-CSFR axis in ex vivo cultured myofibres. On the basis of this, we hypothesized that G-CSF could be important in developing an effective therapy for DMD based on its potential to modulate the supply of multiple stages of regenerated myocytes. It is surprising that haploinsufficiency of G-CSFR leads to early lethality in DMD model mice, indicating that dose reduction of the G-CSF/G-CSFR system could not support the balance between degeneration and regeneration. We also showed that G-CSF administration in mice with severe DMD markedly improves the phenotype, including muscle pathology, functioning and lifespan. Together, our data suggested that the G-CSF-G-CSFR axis is fundamentally important for long-term muscle regeneration, functional maintenance and lifespan extension in DMD mouse models with varying severities.

\section{Results}

G-CSFR is expressed in activated SCs. To explore the precise expression pattern of G-CSFR in skeletal muscle, we examined the SCs on isolated myofibres from the extensor digitorum longus (EDL) muscles of wild-type mice. This model preserves the physiological behaviour of SCs through activation, proliferation and differentiation on myofibres. In the isolated myofibres, PAX7 + quiescent SCs showed no G-CSFR expression by immunostaining at the time of myofibre isolation (Fig. 1a). G-CSFR expression was observed in the PAX7 + cells after $6 \mathrm{~h}$ (Fig. 1a,c), when MYOD expression also appeared (Fig. 1b; Supplementary Fig. 1a). G-CSFR expression was still apparent at $48 \mathrm{~h}$ in the MYOGENIN + SCs, which are committed to differentiation (Supplementary Fig. 1b,c) ${ }^{18}$. By 24 and 48 h, the number of G-CSFR-expressing cells increased to $\sim 94 \%$ of PAX7 + cells (Fig. 1c).

Asymmetrically expressed G-CSFR in activated SCs. Unexpectedly, we found that G-CSFR is asymmetrically distributed within migrating SCs obtained from isolated myofibres at day 5 (Fig. 2a). This asymmetric distribution of G-CSFR was apparent in $\sim 20 \%$ of PAX7 + SCs at $72 \mathrm{~h}$ (Fig. 2b). Syndecan -4 (SDC4) was expressed in the activated SCs until mature myocyte fusion occurred; thus, paired SDC4 + cells were characterized as the daughter cells of SC division ${ }^{19,20}$. The majority of paired SDC4 + cells showed equivalent expression of G-CSFR $(94.4 \%$; Fig. 2c), but in the remaining $5.6 \%$ of paired SDC4 + cells, G-CSFR was detectable in only one daughter cell (Fig. 2d). Similarly, single SDC4 + SCs either express G-CSFR or not (Fig. 2e,f). In $\sim 5 \%$ of paired $\mathrm{PAX} 7+/ \mathrm{SDC} 4+$ cells, one daughter cell highly expressed G-CSFR; in the other daughter cell, G-CSFR expression was not observed (Fig. 2g). In addition, in $\sim 5 \%$ of paired PAX7 + cells, one G-CSFR + daughter cell highly expressed MYOD, whereas no MYOD expression observed in the other (Fig. 2h). These data suggested that G-CSFR is expressed from the activation of SCs through asymmetrical distribution during the first divisions but is not expressed in the self-renewing PAX7 + /MYOD - SCs (Supplementary Fig. 2).

G-CSF increases SCs via the G-CSF-G-CSFR axis. To elucidate whether G-CSFR signalling affects the populations of SCs, we examined the PAX7 + cell population in cultured myofibres from wild-type and G-CSFR-mutated $\left(c s f 3 r^{-1-}\right)$ mice. At 0 and $24 \mathrm{~h}$, there was no significant difference in the number of PAX7 + cells between wild-type and $c s f 3 r^{-/}$mice; however, at $48 \mathrm{~h}$, the csf $3 r-1-$ mice showed a significantly smaller number of PAX7 + cells than that of wild-type mice (Fig. 3a,b). At $24 \mathrm{~h}$, most PAX7 + cells express MYOD, are activated and thereafter proliferate and commit to differentiation ${ }^{21-23}$. In the csf $3 r-/-$ mice, the number of MYOD + /PAX7 + cells was significantly decreased in PAX7 + cells activated for $24 \mathrm{~h}$ (Fig. 3c,d), suggesting that the genetic ablation of G-CSFR signalling negatively affects the population of activated SCs. We then examined the direct role of G-CSF on SCs from wild-type mice. At $72 \mathrm{~h}$, the subpopulation of activated SCs had lost MYOD expression in vivo and on cultured myofibres ${ }^{21-23}$. Surprisingly, 

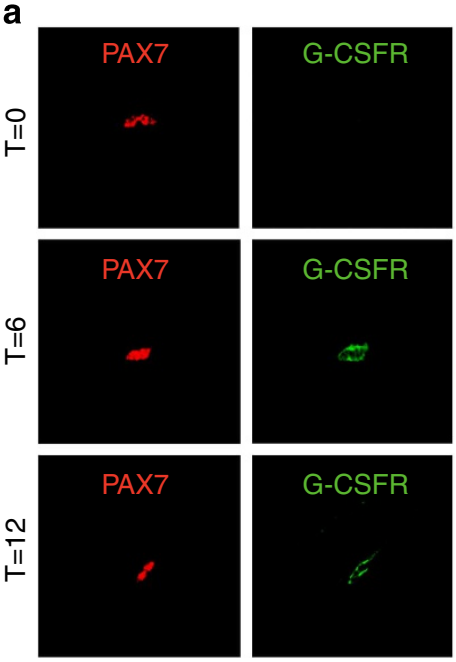

b
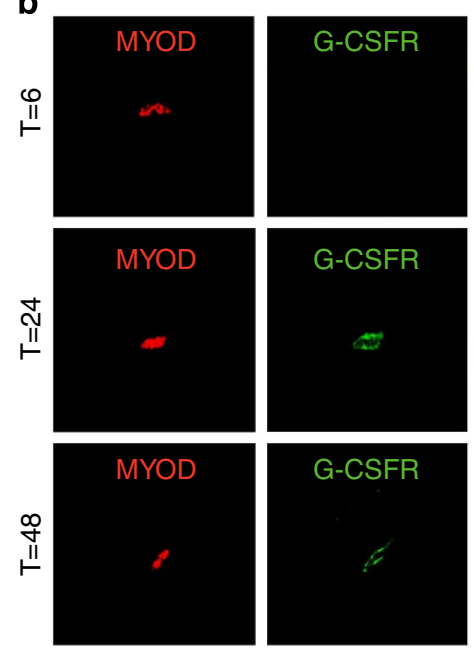
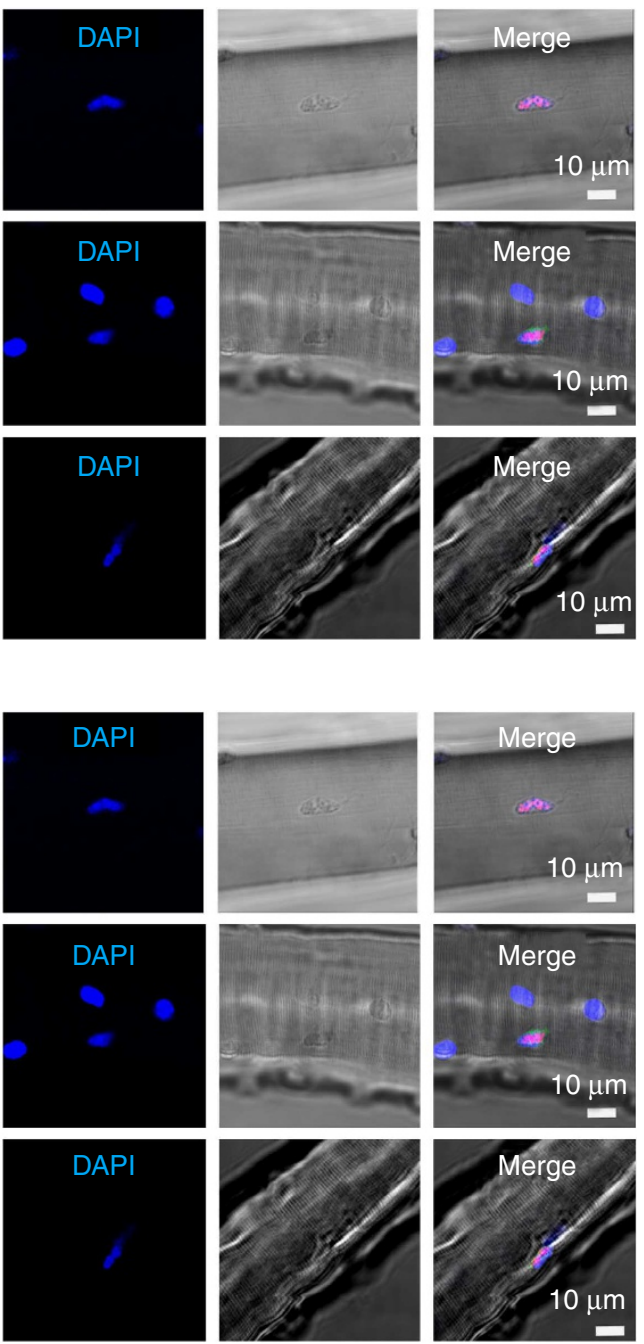

c

G-CSFR+/PAX7+ cell ratio

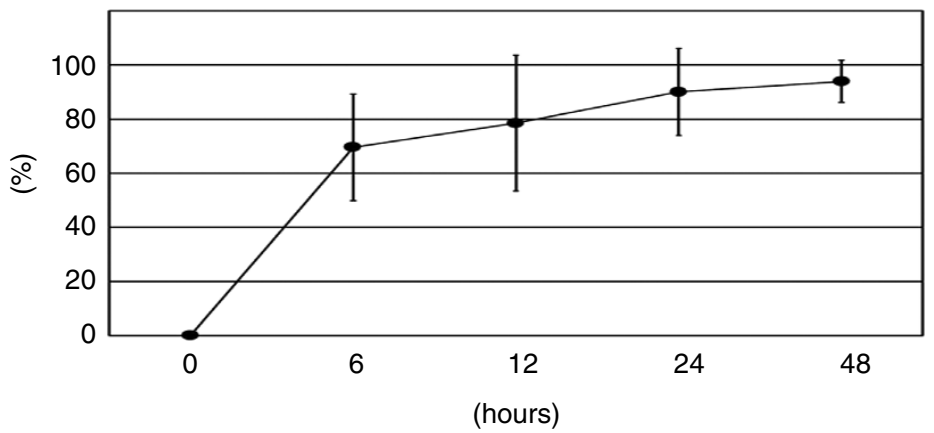

Figure 1 | G-CSFR is expressed in activated satellite cells. (a) Immunostaining of satellite cells on myofibres isolated from EDL muscles for PAX7 (red) and G-CSFR (green), including nuclei stained with DAPI (blue) and bright-field images, at $0 \mathrm{~h}(T=0), 6 \mathrm{~h}(T=6)$ and $12 \mathrm{~h}(T=12)$. Scale bar, $10 \mu \mathrm{m}$. (b) Immunostaining of satellite cells on myofibres for MYOD (red) and G-CSFR (green) at $6 \mathrm{~h}(T=6), 24 \mathrm{~h}(T=24)$ and $48 \mathrm{~h}(T=48)$. Scale bar, $10 \mu \mathrm{m}$. (c) The G-CSFR + /PAX7 + cell ratio at 0, 6, 12, 24 and 48 h ( $T=0: n=53 ; T=6: n=73 ; T=12: n=45 ; T=24: n=41 ; T=48: n=297 ;$ each myofibre: $n=20$-30). All myofibres were obtained from $\sim 10$-week-old wild-type mice. Data are shown as mean \pm s.d. DAPI, 4',6-diamidino-2-phenylindole dihydrochloride.

G-CSF significantly increased the population of SCs throughout the differentiation stages, including self-renewal SCs, as shown by the $\mathrm{PAX} 7+/ \mathrm{MYOD}-$ cells at $72 \mathrm{~h}$ in wild-type mice; in addition, csf $3 r-/-$ mice showed significantly decreased numbers of SCs throughout the differentiation stages (Fig. 3e,f; Supplementary Table 1). To elucidate the effect of G-CSF on the population of PAX7 + cells in vivo, we examined SC behaviour in a cardiotoxin injury model (Fig. $3 g$ ). In the $c s f 3 r-/-$ mice, the population of PAX7 +-expressing cells was significantly decreased at 14 days after cardiotoxin injection (Fig. 3h,i), suggesting that genetic G-CSFR signal ablation negatively affects the population of PAX7 + cells in vivo. 
a
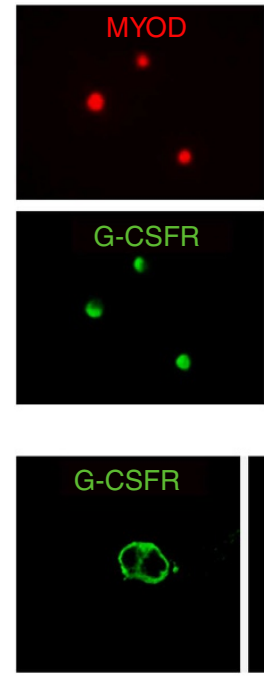

d

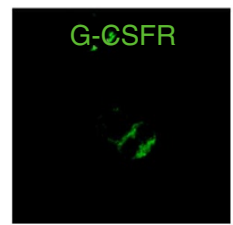

$\mathbf{e}$

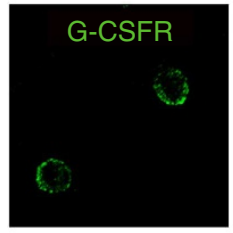

$\mathbf{f}$

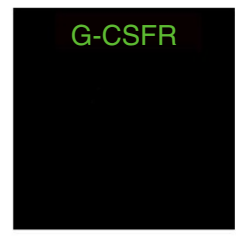

g

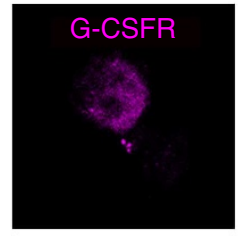

h

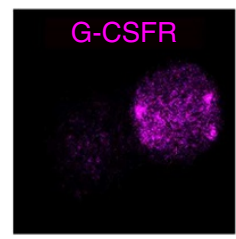

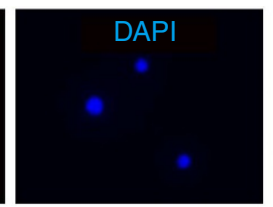

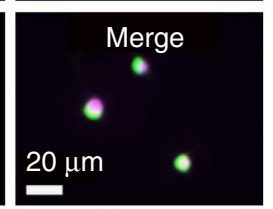

b
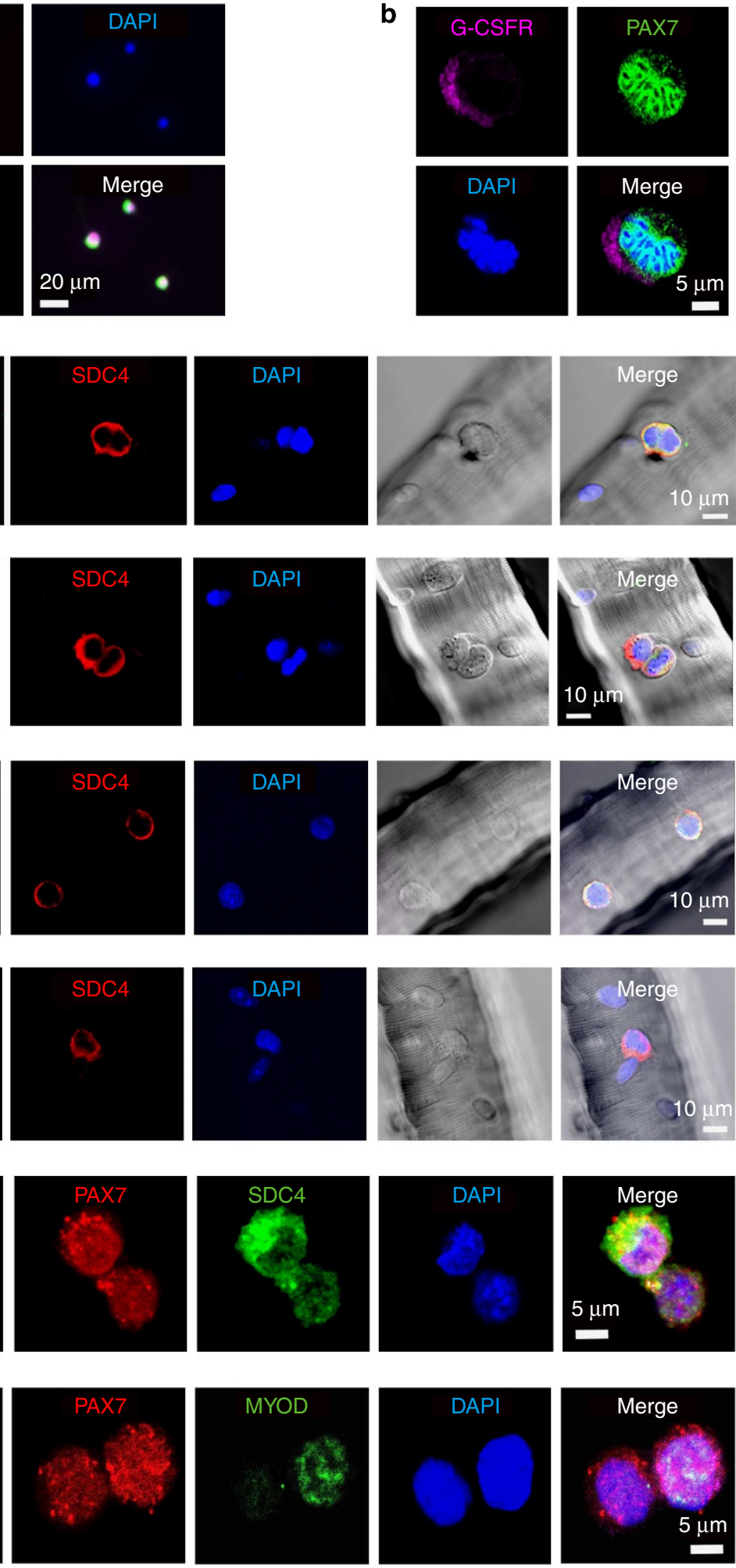

Figure 2 | G-CSFR is asymmetrically expressed in activated satellite cells. (a) Immunostaining of satellite cells for MYOD (red), G-CSFR (green) and DAPI (blue) at day 5. Scale bar, $20 \mu \mathrm{m}$. (b) Immunostaining of satellite cells for G-CSFR (purple), PAX7 (green) and DAPI (blue) at day 3. Scale bar, $5 \mu \mathrm{m}$. (c) Immunostaining of dividing satellite cells on myofibres for G-CSFR (green), SDC4 (red) and DAPI (blue), as well as bright-field (BF) images, at $48 \mathrm{~h}$ (satellite cells with symmetric G-CSFR expression: $n=51$; satellite cells with asymmetric expression: $n=3$ ). Scale bar, $10 \mu \mathrm{m}$. (d) Immunostaining of dividing satellite cells on myofibres for G-CSFR (green), SDC4 (red) and DAPI (blue), as well as BF images, at $48 \mathrm{~h}$. Scale bar, $10 \mu \mathrm{m}$. (e,f) Immunostaining of satellite cells on myofibres for SDC4 (red), G-CSFR (green) and DAPI (blue), as well as BF images, at $48 \mathrm{~h}$. Scale bar, $10 \mu \mathrm{m}$. (g) Immunostaining of cultured dividing satellite cells for G-CSFR (purple), PAX7 (red), SDC4 (green) and DAPI (blue). Scale bar, $5 \mu \mathrm{m}$. (h) Immunostaining of cultured dividing satellite cells for G-CSFR (purple), PAX7 (red), MYOD (green) and DAPI (blue) at day 3 (among 273 dividing satellite cells, dividing satellite cells with symmetric G-CSFR expression: $n=5$; dividing satellite cells with asymmetric expression: $n=268$ ). Scale bar, $5 \mu$ m. All myofibres were obtained from 10-week-old wild-type mice. DAPI, 4',6-diamidino-2-phenylindole dihydrochloride. 

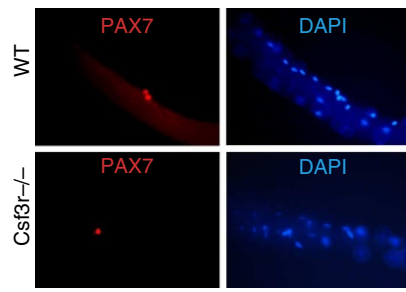

WT

C

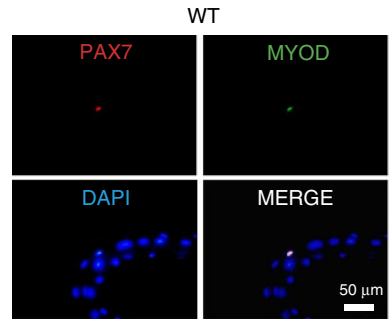

e

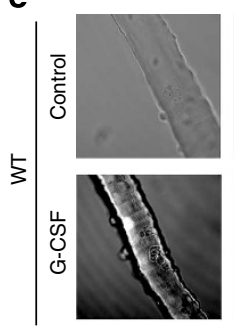

g
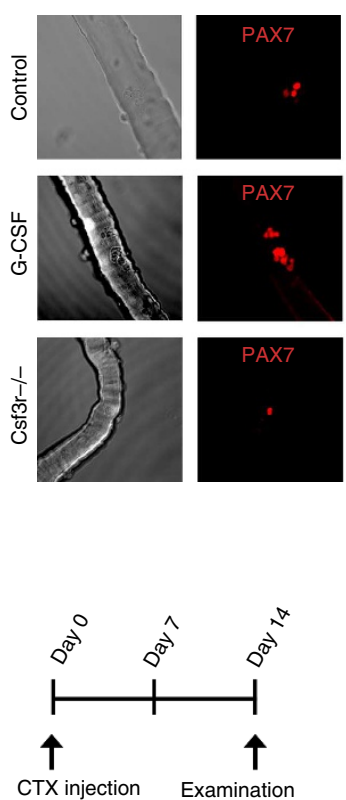
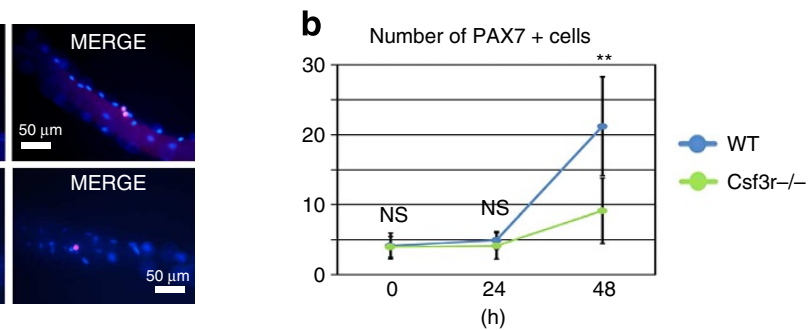

(h)

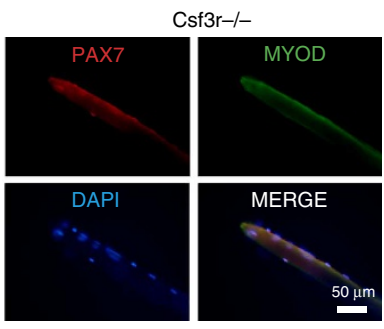

d

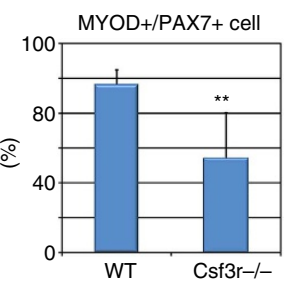

f
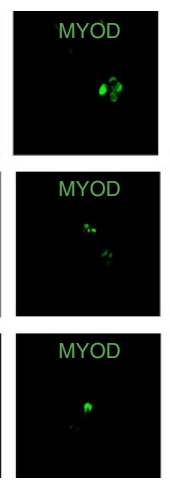

h

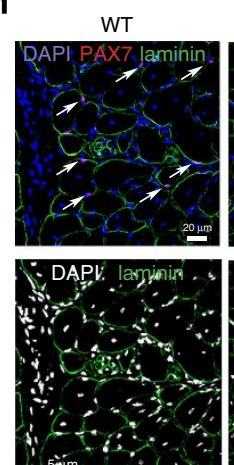

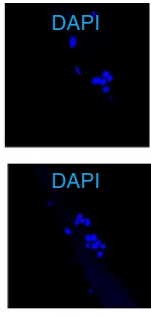
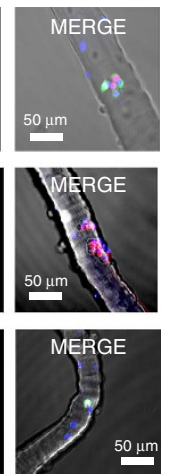

Csf3r-1-

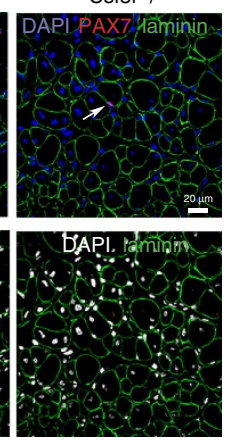

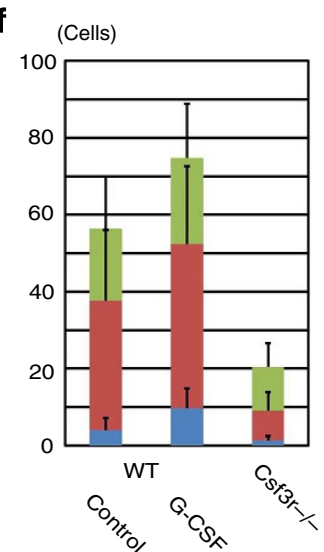

- $\operatorname{PAX7}(-), \operatorname{MYOD}(+)$

- $\operatorname{PAX7}(+), \operatorname{MYOD}(+)$

- $\operatorname{PAX} 7(+), \operatorname{MYOD}(-)$

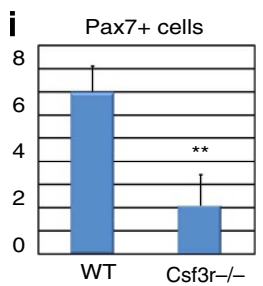

Figure 3 | G-CSF increases satellite cells in multiple differentiation stages. (a) Immunostaining of satellite cells on myofibres of $\sim 10$-week-old WT and csf3r-/- mice for PAX7 (red) and DAPI (blue) at $48 \mathrm{~h}$. Scale bar, $50 \mu \mathrm{m}$. (b) Time course of the number of satellite cells (PAX7 +) at 0,24 and $48 \mathrm{~h}$ per myofibre of WT and $c s f 3 r-/-$ mice. Data are shown as mean \pm s.d. ${ }^{\star \star} P<0.01$; equal variance Student's $t$-test. Each fibre number: $n=20-29$.

(c) Immunostaining of satellite cells on myofibres of WT and csf3r - / - mice for PAX7 (red), MYOD (green) and DAPI (blue) at $24 \mathrm{~h}$. Scale bar, $50 \mu \mathrm{m}$. (d) The percentage of MYOD $+/$ PAX7 + satellite cells at $24 \mathrm{~h}$. Data are shown as mean \pm s.d. ${ }^{\star \star} P<0.01$; unequal variance Student's $t$-test. Each fibre number: $n=29$ ). (e) Immunostaining of satellite cells on myofibres of WT mice administered control and G-CSF and csf3r $-/-$ mice after being cultured for $72 \mathrm{~h}$ for PAX7 (red), MYOD (green) and DAPI (blue), as well as bright-field images. Scale bar, $50 \mu$ m. (f) Quantification of the number of PAX7 - /MYOD + (green), PAX7 + /MYOD + (red) or PAX7 + /MYOD - (blue) satellite cells per myofibre. Data are shown as mean \pm s.d. One-way analysis of variance with Bonferroni's post hoc test. Quantification information in Supplementary Table 1. (g) Time course of cardiotoxin (CTX) injection and examination for 8-week-old WT and csf3r-/ - mice. (h) Immunostaining of cross-sections of TA muscle of WT and csf3r - / - mice at 14 days after CTX injection for laminin, PAX7 and DAPI. Upper panel: merged with laminin (green), PAX7 (red) and DAPI (blue). Lower panel: merged with laminin (green) and DAPI (white). Arrows indicate PAX7 + /DAPI + cells. Scale bar, $20 \mu \mathrm{m}$. (i) Number of PAX7 + /DAPI + cells per 100 myocytes of WT and $c s f 3 r-/-$ mice. Data are shown as mean \pm s.d. ${ }^{\star}{ }^{\star} P<0.01$; unequal variance Student's $t$-test. $n=5-6$ per group. DAPI, 4',6-diamidino-2-phenylindole dihydrochloride; WT, wild type. 

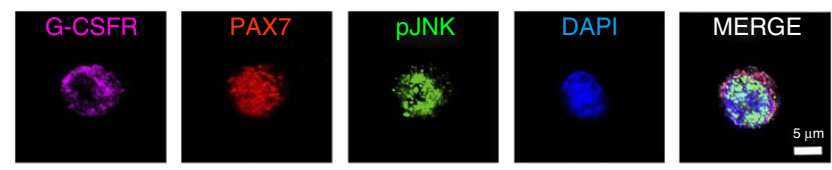

b
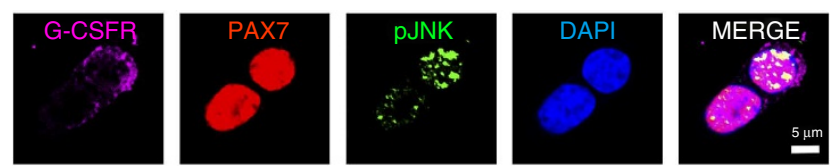

C
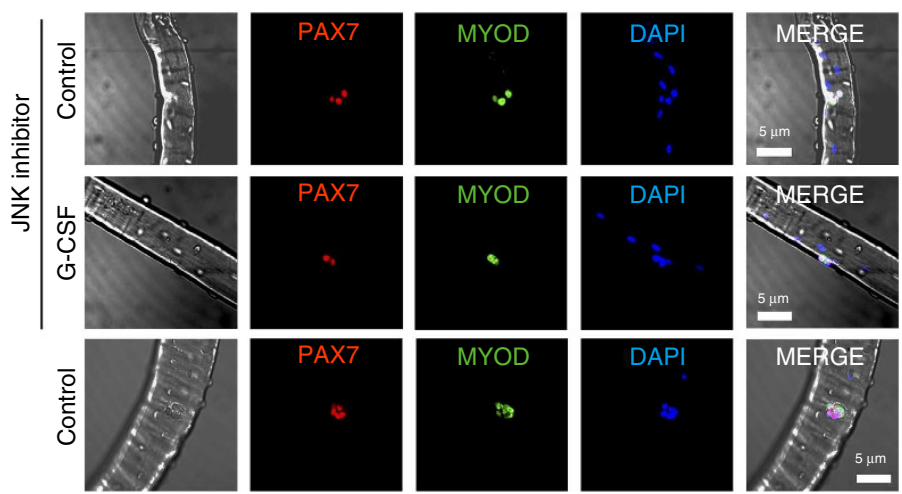

\section{d}

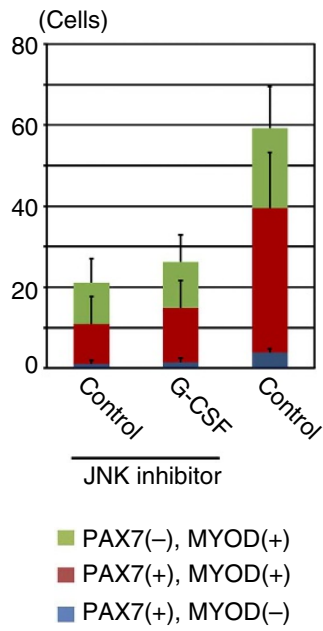

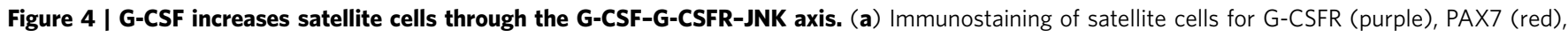
pJNK (green) and DAPI (blue) at day 3. Scale bar, $5 \mu \mathrm{m}$. (b) Immunostaining of dividing satellite cells for G-CSFR (purple), PAX7 (red), pJNK (green) and DAPI (blue) at day 3. Scale bar, $5 \mu \mathrm{m}$. (c) Immunostaining of satellite cells on myofibres of WT mice for PAX7 (red), MYOD (green) and DAPI (blue), as well as bright-field images, following JNK inhibitor administration with or without G-CSF at $72 \mathrm{~h}$. Scale bar, $50 \mu \mathrm{m}$. (d) Quantification of the number of PAX7 - /MYOD + (green), PAX7 + /MYOD + (red) or PAX7 + /MYOD - (blue) satellite cells per myofibre. Data are shown as mean \pm s.d. One-way analysis of variance with Bonferroni's post hoc test. Quantification information in Supplementary Table 1. DAPI, 4',6-diamidino-2-phenylindole dihydrochloride.

We previously showed that G-CSF apparently activates JNK and p38 signalling in cultured myoblasts 9 . Therefore, we examined whether these pathways are active in activated SCs. Immunostaining for pJNK and pp38 revealed that both JNK and p38 are activated in G-CSFR + SCs (Fig. 4a; Supplementary Fig. 3a). The p38 pathway is required for SC activation and MYOD induction following proliferation ${ }^{24}$; herein, p38 inhibition in single myofibres decreased the population of $\mathrm{PAX} 7-1$ MYOD + SCs with or without G-CSF (Supplementary Fig. 3b,c; Supplementary Table 1). Although JNK signalling is involved in myoblast differentiation ${ }^{25}$, its role in SC behaviour remains unclear. In paired PAX7 + cells, the G-CSFR-expressing daughter cells highly expressed activated JNK, whereas the other daughter cells without G-CSFR expression did not (Fig. 4b). JNK inhibition also strongly reduced the number of $\mathrm{PAX} 7+1$ MYOD - and PAX7 + /MYOD + cells in single myofibres (Fig. 4c,d; Supplementary Table 1). These data indicated that G-CSF increases both activated and self-renewal SCs through the G-CSF-G-CSFR-JNK axis, suggesting that G-CSF could increase long-term regeneration in chronic skeletal muscle injury through maintenance of the SC pool.

G-CSF increases muscle regeneration in $m d x$ mice. Patients with DMD show progressive physical impairment leading to the death by $20-30$ years of age, whereas a mouse model of DMD $(m d x$ mice) harbouring a dystrophin mutation has minimal physical impairment and a normal lifespan ${ }^{26}$. Indeed, $m d x$ mice appear grossly normal (Supplementary Fig. 4a). However, pathological analysis revealed apparent muscle degeneration and regeneration in these $m d x$ mice from 3 to 5 weeks of age
(Supplementary Fig. 4b). Myocyte numbers in the tibialis anterior (TA) muscle were decreased at 3 weeks of age, but were thereafter recovered (Supplementary Fig. 4c). Generally, healthy myocytes have peripherally located nuclei, and regenerating and regenerated myocytes have central nuclei. The $m d x$ mice myocytes showed central nuclei from 4 weeks of age and increased in number up to 12 weeks of age (Supplementary Fig. 4d). These data suggested initial injury to the muscle fibres with subsequent regeneration to an almost normal state in the $m d x$ mice. The histological observation of TA muscle showed strong G-CSFR expression in regenerating myocytes (Supplementary Fig. 4e), implicating the possible role of $\mathrm{G}-\mathrm{CSF}$ in the muscle regeneration process. We then examined whether G-CSF administration would improve skeletal muscle regeneration in $m d x$ mice. We examined the gross appearance and functional differences following daily intraperitoneal (i.p.) G-CSF injections from 3 to 5 weeks of age (Fig. 5a). Immunostaining for laminin showed the typically fine cellular architecture of TA muscle (Fig. 5b). The number of myocytes with central nuclei significantly increased with G-CSF administration at 5 weeks of age (Fig. 5 c). The BF-45 antibody recognizes myosin heavy chain present during embryonic and neonatal skeletal muscle development and in newly formed adult regenerating myocytes ${ }^{27}$. Immunostaining for BF-45 in 4 -weekold mice treated with G-CSF revealed significantly increased numbers of regenerating myocytes (Fig. 5d,e). Measuring the individual cross-sectional area of the myocytes further revealed a significantly increased proportion of small myocytes, indicating a substantial amount of newly formed regenerated myocytes at 5 weeks of age (Fig. 5f). Given that muscle size is a predictive value for muscle function, the demonstrated increase in the TA muscle 
a

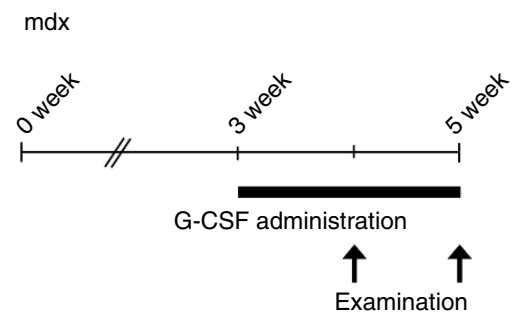

d

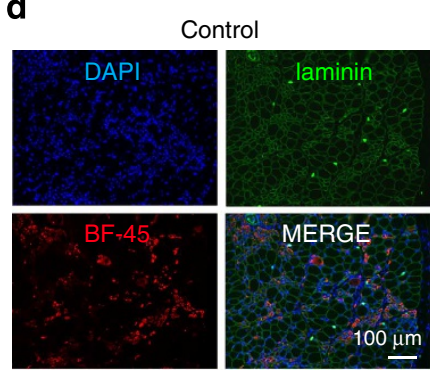

f

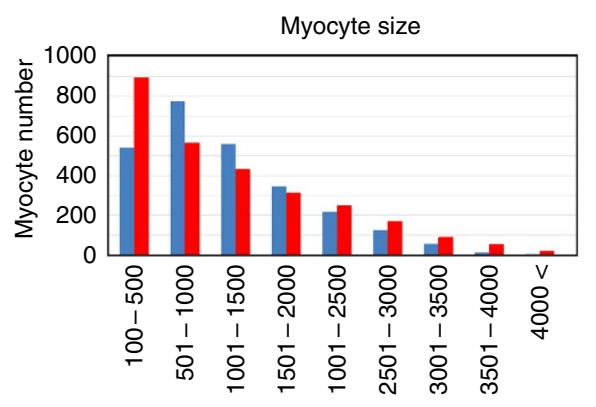

$\left(\mu \mathrm{m}^{2}\right)$ b
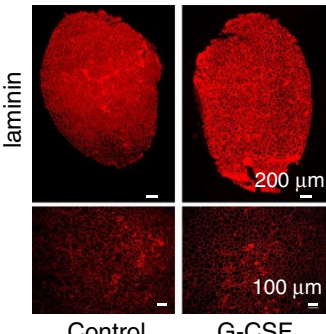

Control

G-CSF

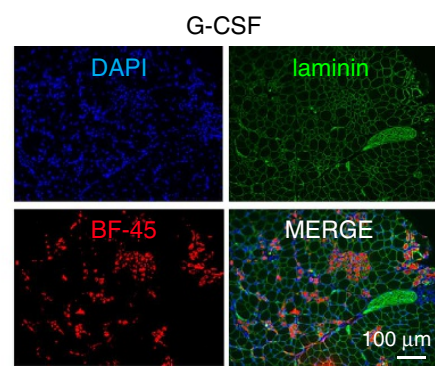

g
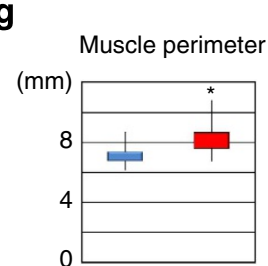

h

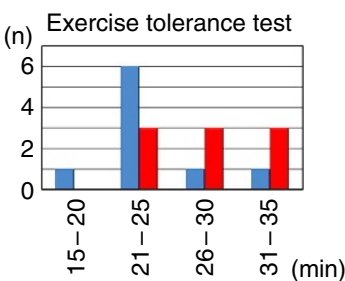

c

Central

nuclear myocytes

(\%)

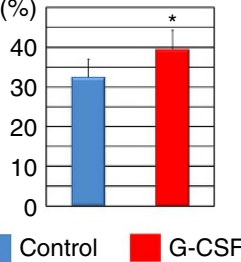

Control G-CSF

e

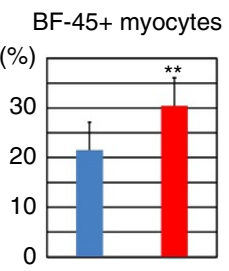

i

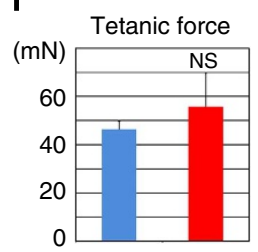

j

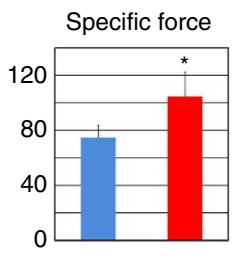

Figure 5 | G-CSF increases skeletal muscle regeneration in $\mathbf{m d x}$ mice. (a) Time course of G-CSF administration and examination in $m d x$ mice. (b) Immunostaining of cross-sections of TA muscle for laminin at 5 weeks of age mdx mice. Scale bar, $200 \mu \mathrm{m}$ (upper panel); $100 \mu \mathrm{m}$ (lower panel).

(c) The percentages of myocytes with central nuclei in cross-sections of TA muscle at 5 weeks of age. Data are shown as mean \pm s.d. ${ }^{\star} P<0.05$; equal variance Student's t-test. $n=8$ per group. (d) Immunostaining of cross-sections of TA muscle for BF-45 (red), laminin (green) and DAPI (blue) at 4 weeks of age. Scale bar, $100 \mu \mathrm{m}$. (e) The percentages of BF-45-positive myocytes in cross-sections of TA muscle at 4 weeks of age. Data are shown as mean \pm s.d. ${ }^{\star \star} P<0.01$; unequal variance Student's $t$-test. $n=5$ per group. (f) The individual fibre cross-sectional area of individual myocytes in cross-sections of TA muscles at 5 weeks of age. Data are shown as mean. $n=6$ per group. ( $\mathbf{g}$ ) Muscle perimeters of cross-sections of TA muscles at 5 weeks of age. Data are shown as box plot. Top, maximum; bottom, minimum. ${ }^{\star} P<0.05$; equal variance Student's $t$-test. $n=8$ per group. (h) Exercise tolerance test results at 5 weeks of age. $n=9$ per group. (i) Tetanic force examination of ex vivo EDL muscle tension at 5 weeks of age. Data are shown as mean \pm s.d. No significant difference; unequal variance Student's $t$-test; $n=6$ per group. (j) Specific forces, which are the ratios of tetanic force to muscle size, measured at 5 weeks of age. Data are shown as mean \pm s.d. ${ }^{\star} P<0.05$; unequal variance Student's $t$-test. $n=5$ per group. DAPI, 4',6-diamidino-2-phenylindole dihydrochloride; NS, not significant.

perimeter in G-CSF-treated mice at 5 weeks of age suggested enhanced muscle function recovery (Fig. 5g). G-CSF also significantly improved exercise capacity in the mice by 5 weeks of age based on in vivo exercise tolerance testing (Fig. 5h). In a pure muscle function test, ex vivo tetanic force measurements and specific force examinations showed significantly improved muscle tension in the treated mice at 5 weeks of age (Fig. $5 \mathrm{i}, \mathrm{j}$ ).

Next, we examined whether G-CSF treatment would accelerate the regeneration process following cardiotoxin injury in $m d x$ mice (Fig. 6a). The number of myocytes with central nuclei significantly increased by G-CSF administration in cardiotoxininjured $m d x$ mice (Fig. 6b,c). The number of BF-45 + myocytes was also significantly increased by G-CSF treatment (Fig. 6d,e). The TA muscle perimeter was also increased by G-CSF treatment in cardiotoxin-injured $m d x$ mice (Fig. 6f). These data suggested that G-CSF significantly improves muscle function through myocyte regeneration in $m d x$ mice.

Haploinsufficiency of G-CSFR induces lethality in $m d x$ mice. To clarify the roles of G-CSF in DMD, we next used csf3r-1mice, which show a slight haematological disorder and a normal lifespan ${ }^{28,29}$. We hypothesized that breeding G-CSFR homozygote-knockout $m d x$ mice $\left(m d x / c s f 3 r^{-/-}\right)$would produce 
mice with severely impaired muscle regeneration and function. Surprisingly, G-CSFR heterozygote $\mathrm{mdx}\left(m d x / c s f 3 r^{+/-}\right)$mice showed early partial lethality after birth and late partial lethality at 3-5 weeks of age (Fig. 7a). These striking data indicated the importance of proper G-CSF signalling in DMD. The $m d x, c s f 3 r^{+1-}$ and $m d x / c s f 3 r^{+1-}$ mice were of normal gross a

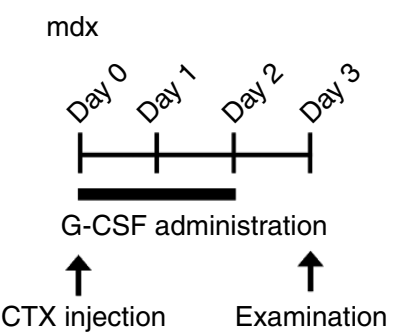

b

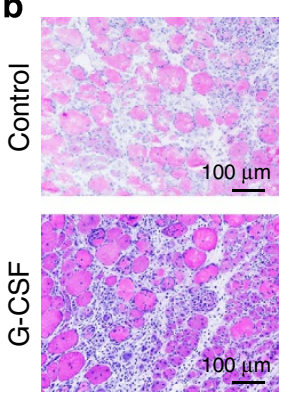

C

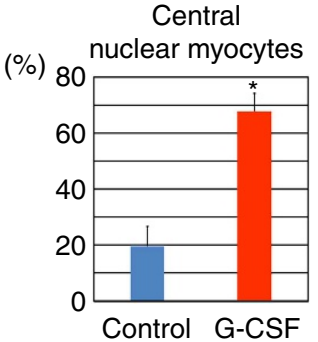

d

Control
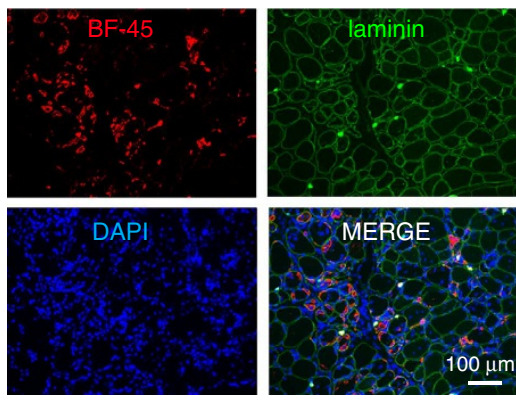

e

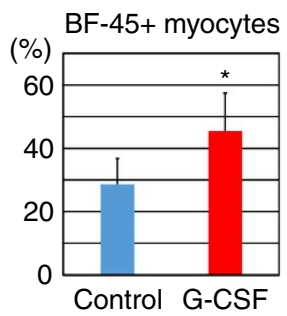

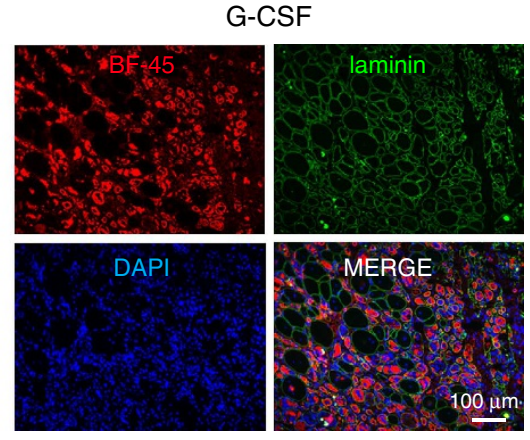

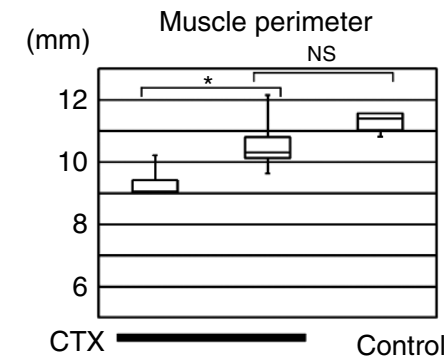

Figure 6 | G-CSF increases muscle regeneration in $\mathbf{m d x}$ mice after CTX injury. (a) Time course of CTX and G-CSF administration, and examination in 8-week-old $m d x$ mice. (b) Haematoxylin and eosin staining of cross-sections of TA muscle at day 3 after CTX injection. Scale bar, $100 \mu \mathrm{m}$. (c) The percentages of central nuclear myocytes in cross-sections of TA muscle at day 3 after CTX injection. Data are shown as mean \pm s.d. ${ }^{\star} P<0.05$; unequal variance Student's $t$-test. $n=4$ per group. (d) Immunostaining of cross-sections of TA muscle for BF-45 (red), laminin (green) and DAPI (blue) at day 3 after CTX injection. Scale bar, $100 \mu \mathrm{m}$. (e) The percentages of BF-45-positive myocytes in cross-sections of TA muscle at day 3 after CTX injection. Data are shown as mean \pm s.d. ${ }^{\star} P<0.05$; unequal variance Student's $t$-test. $n=5$ per group. (f) Muscle perimeters of cross-sections of TA muscle at day 3 after CTX injection. Data are shown as box plot. Top, maximum; bottom, minimum. ${ }^{\star} P<0.05$; Student's $t$-test. $n=4-6$ per group. $C T X$, cardiotoxin; DAPI, 4',6-diamidino-2-phenylindole dihydrochloride; NS, not significant.

Figure 7 | G-CSF signalling is essential for the survival in $\mathbf{m d x}$ mice. (a) Kaplan-Meier survival curve of $c s f 3 r+/-($ red, $n=50), m d x(y e l l o w, n=50)$ and $m d x / c s f 3 r^{+/-}$(blue, $n=53$ ) mice. (b) Gross appearance of $m d x, c s f 3 r+/-$, $m d x / c s f 3 r^{+/-}$and WT mice at post-natal day 1. (c) Body weights of $m d x(n=7), c s f 3 r^{+/-}(n=9), m d x / c s f 3 r+/-(n=11)$ and WT $(n=15)$ mice at post-natal day 1 . Data are shown as box plot. Top, maximum; bottom, minimum. ${ }^{\star \star} P<0.01$; unequal variance Student's $t$-test. (d) Evans blue staining of the diaphragm of $m d x, c s f 3 r^{+} /-$, $m d x / c s f 3 r+/-$ and WT mice at postnatal day 1. (e) Haematoxylin and eosin staining of the diaphragm of $m d x, c s f 3 r^{+/}-, m d x / c s f 3 r^{+/}-$and WT mice at post-natal day 1. Scale bar, $50 \mu m$. (f) Diaphragm thickness of $m d x, c s f 3 r^{+/-}, m d x / c s f 3 r+/-$ and WT mice at post-natal day 1 ( $n=5$ per group). Data are shown as mean \pm s.d. ${ }^{\star \star} P<0.01$; Student's t-test; $n=5$ per group. (g) Immunostaining of cross-sections of the diaphragm for PAX7 (red), laminin (green) and DAPI (blue) at post-natal day 1 of $m d x, c s f 3 r^{+/-}, m d x / c s f 3 r^{+/-}$and WT mice. Scale bar, $50 \mu \mathrm{m}$. (h) Number of PAX7 +/DAPI + cells per 100 myocytes at post-natal day 1 of $m d x$, $c s f 3 r^{+/-}, m d x / c s f 3 r+/-$ and WT mice. Data are shown as mean \pm s.d. ${ }^{\star \star} P<0.01$; Student's $t$-test; $n=5$ per group. (i) Whole-body plethysmography to examine the respiratory function of individual mice. (j) Breath per min (BPM) in 8-week-old $m d x$ and $m d x / c s f 3 r+/-$ mice. Data are shown as mean \pm s.d. No significant difference; unequal variance Student's $t$-test; $n=4$ per group. (k) Tidal volume of 8-week-old $m d x$ and $m d x / c s f 3 r+/-m i c e . ~ D a t a$ are shown as mean \pm s.d. No significant difference; unequal variance Student's $t$-test; $n=4$ per group. (I) Body weight growth curves of $c s f 3 r+/-$ and $m d x / c s f 3 r^{+/-}$mice from 2 to 8 weeks of age. Data are shown as mean \pm s.d. ${ }^{\star} P<0.05,{ }^{\star \star} P<0.01 ;$ unequal variance Student's $t$-test; $n=7$ per group. DAPI, 4',6-diamidino-2-phenylindole dihydrochloride; NS, not significant; WT, wild type. 
appearance at post-natal day (P) P1 (Fig. 7b); however, $m d x / c s f 3 r^{+1-}$ mice had significantly reduced body weights compared with $m d x$ littermates (Fig. 7c). To elucidate the cause of early lethality, we examined the mice for gross appearance of the diaphragm and degenerative lesions by Evans blue staining. Lesions were largely absent from the $m d x, c s f 3 r^{+1-}$ and wild-type mice, whereas the $m d x / c s f 3 r^{+I-}$ mice showed obvious degenerative lesions (Fig. 7d,e). Diaphragm thickness was also significantly decreased in $m d x / c s f 3 r^{+/-}$mice compared with $m d x$ littermates (Fig. $7 \mathrm{f}$ ). The population of PAX7 + cells in diaphragm was also significantly decreased in $m d x / c s f 3 r^{+/-}$mice (Fig. 7g,h). Whole-body plethysmography indicated that $m d x / c s f 3 r+l-$ mice showed mildly impaired respiratory function (Fig. $7 \mathrm{i}-\mathrm{k}$ ). In addition, the surviving

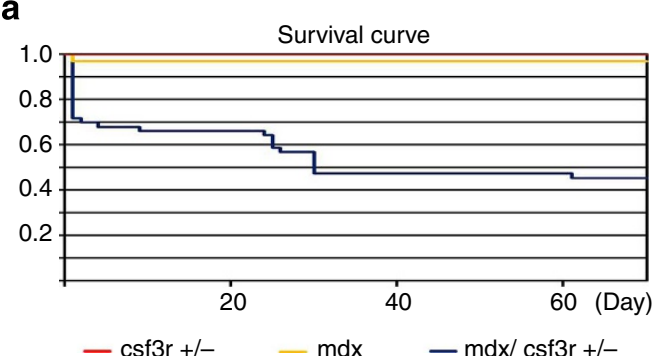

b

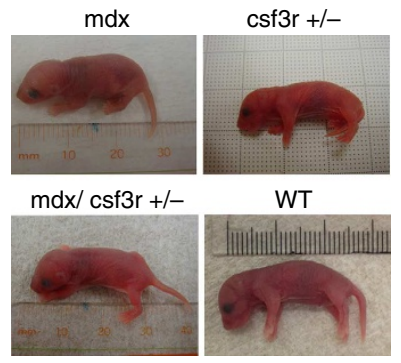

C

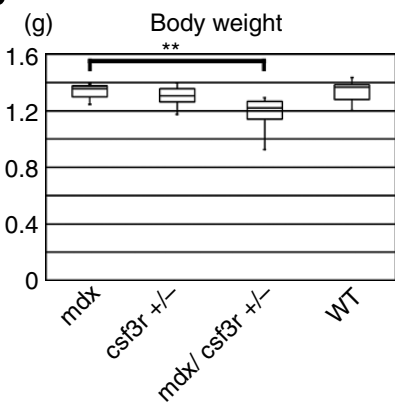

f

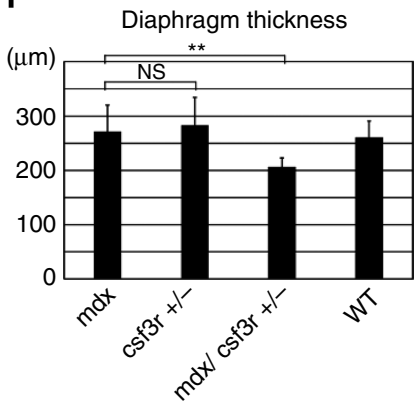

h

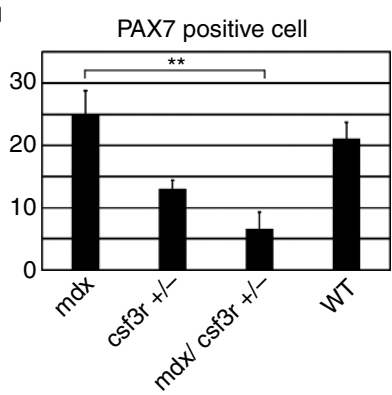

i

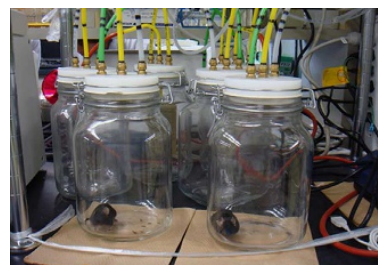

d

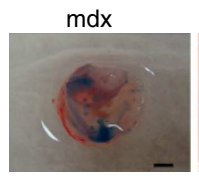

$\operatorname{csf} 3 r+1-$

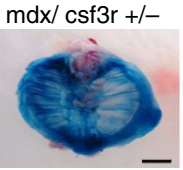

WT
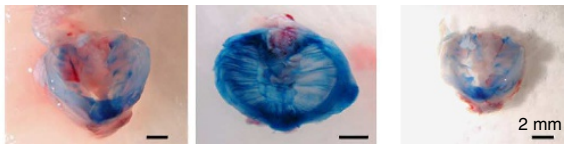

e

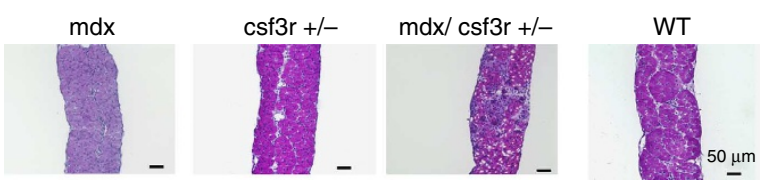

g

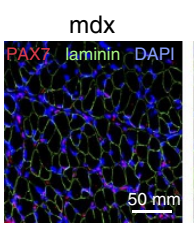
csf3r + - -

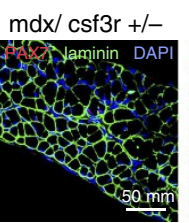
WT
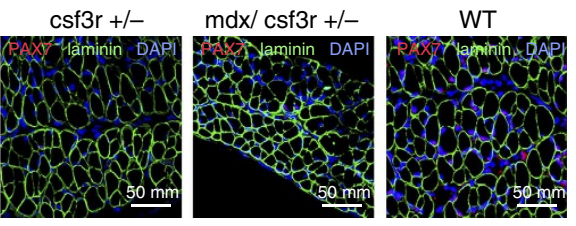

$\mathbf{k}$
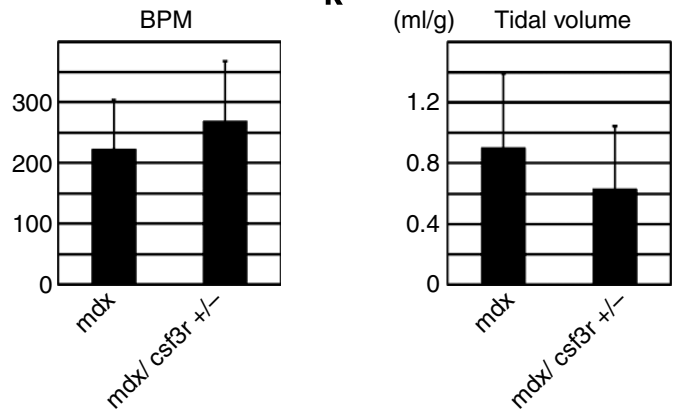

I

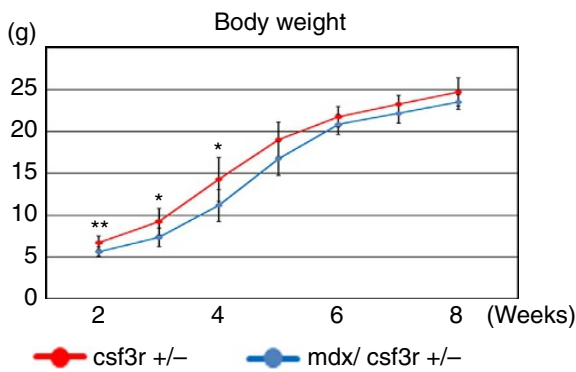


a

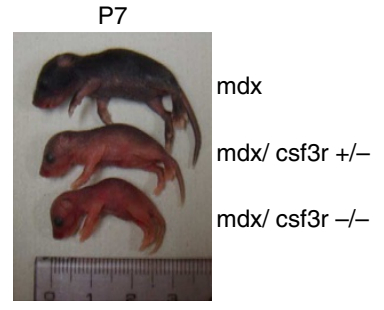

b

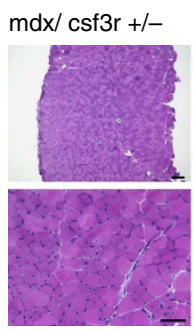

2 weeks

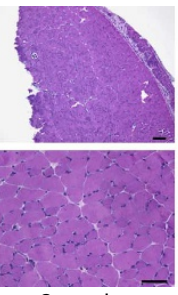

3 weeks
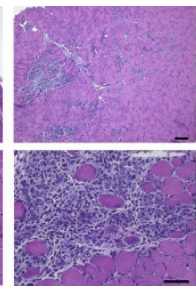

4 weeks
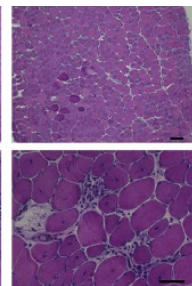

5 weeks

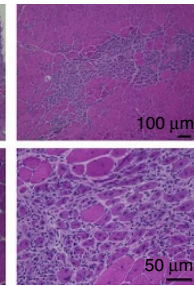

8 weeks c

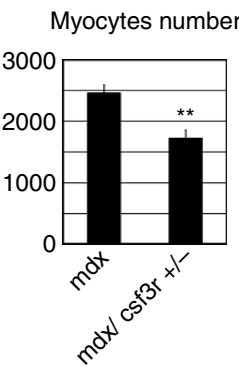

g

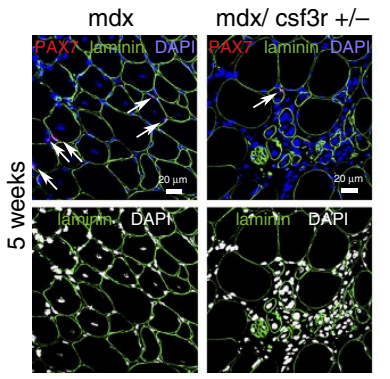

d

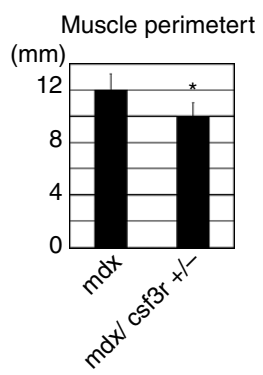

e

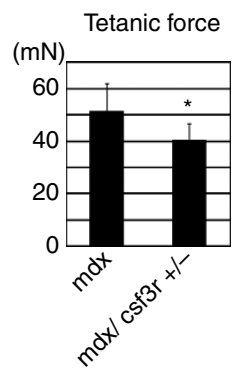

f

Specific force

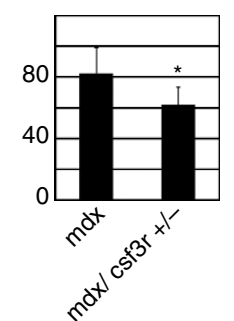

h

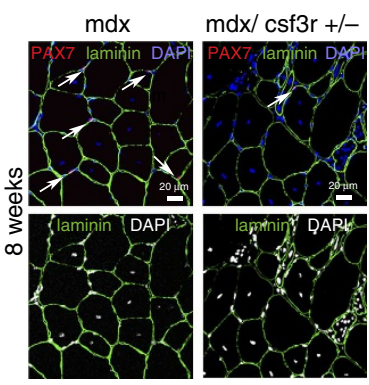

Pax $7+$ cells ( 5 weeks) Pax $7+$ cells ( 8 weeks)
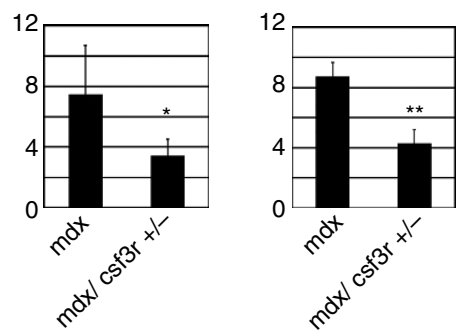

Figure 8 | Haploinsufficiency of G-CSFR reduces muscle regeneration in $\mathbf{m} \boldsymbol{d x}$ mice. (a) Gross appearance analyses showing body size differences among $m d x, m d x / c s f 3 r^{+/-}$and $m d x / c s f 3 r^{-/-}$mice at post-natal day 7 (left panel) and between $m d x / c s f 3 r^{+/}-$and $m d x / c s f 3 r^{-/-}$mice at post-natal day 13 (right panel). (b) Haematoxylin and eosin staining of cross-sections of TA muscle from $\mathrm{mdx} / \mathrm{cs} f 3 \mathrm{r}^{+/}-$mice from 2 to 8 weeks of age are shown in the lower (upper panel) and higher (lower panel) magnified images. Scale bar, $100 \mu \mathrm{m}$ (upper panel); $50 \mu \mathrm{m}$ (lower panel). (c) The total numbers of myocytes in cross-sections of TA muscle at 8 weeks. Data are shown as mean \pm s.d. ${ }^{\star \star} P<0.01$; unequal variance Student's $t$-test; $n=5$ per group. (d) Perimeters of cross-sections of TA muscle at 8 weeks of age. Data are shown as mean \pm s.d. ${ }^{\star} P<0.05$; unequal variance Student's $t$-test; $n=5$ per group. (e) Tetanic force examination of ex vivo EDL muscle tension at 8 weeks of age. Data are shown as mean \pm s.d. ${ }^{\star} P<0.05$; unequal variance Student's $t$-test; $n=5$ per group. (f) Specific forces, represented by tetanic force per muscle size ratios, at 8 weeks of age. Data are shown as mean $\pm \mathrm{s}$.d. ${ }^{\star} P<0.05$; unequal variance Student's $t$-test; $n=6$ per group. (g) Immunostaining of cross-sections of TA muscle from $m d x$ and $m d x / c s f 3 r+/-m i c e$ at 5 and 8 weeks of age for laminin, PAX7 and DAPI. Upper panel: merged with laminin (green), PAX7 (red) and DAPI (blue). Lower panel: merged with laminin (green) and DAPI (white). Arrows indicate PAX7 + /DAPI + cells. Scale bar, $20 \mu \mathrm{m}$. (h) Number of PAX7 + /DAPI + cells per 100 myocytes of $m d x$ and $m d x / c s f 3 r^{+/-}$mice at 5 and 8 weeks of age. Data are shown as mean \pm s.d. ${ }^{\star} P<0.05,{ }^{\star \star} P<0.01$; unequal variance Student's $t$-test; $n=5$ per group. DAPI, 4',6-diamidino-2-phenylindole dihydrochloride; NS, not significant; WT, wild type.

$m d x / c s f 3 r^{+1-}$ mice showed consecutive body weight loss compared with $c s f 3 r^{+/-}$mice (Fig. 7i). Because most $m d x /$ $c s f 3 r^{+1-}$ mice with a strong phenotype died before 5 weeks of age and relative healthy $m d x / c s f 3 r^{+1-}$ mice survive, the differences in body weight and respiratory function would be decreased thereafter.
In $m d x$ mice, skeletal muscle degeneration and regeneration is prominent at 3-5 weeks of age. We therefore proposed that $m d x / c s f 3 r^{+/-}$mice might show deteriorating muscle regeneration and function around that age. Gross examination revealed a smaller body size in $m d x / c s f 3 r^{+1-}$ and $m d x / c s f 3 r^{-1-}$ mice at P7, and apparently smaller body size in $m d x / c s f 3 r^{-1-}$ mice at 
P13 (Fig. 8a). $M d x / c s f 3 r^{+/-}$mice that survived the initial early lethality prevalence showed significantly increased degeneration with decreased numbers of regenerating myocytes at $\sim 4$ weeks of age that remained at $\sim 8$ weeks of age (Fig. $8 \mathrm{~b}$ ). The myocyte number and TA muscle perimeter were also significantly decreased in $m d x / c s f 3 r^{+1-}$ mice at 8 weeks of age (Fig. $8 c, d$ ). Ex vivo muscle tension examination also indicated significantly decreased tetanic and specific forces in the $m d x / c s f 3 r+1-$ mice compared with $m d x$ mice (Fig. 8e,f). The population of PAX7 + cells was also significantly decreased in $m d x / c s f 3 r^{+/-}$mice (Fig. 8g,h). These results confirmed that G-CSF signal is crucial for skeletal muscle regeneration in $m d x$ mice.
G-CSF supports the survival of severe DMD model mice. The physically normal appearance of $m d x$ mice could reflect a redundant function of the utrophin gene, a dystrophin paralogue. The $m d x / u_{t r n}{ }^{-1-}$ mice that express neither dystrophin nor utrophin manifest severe dystrophy resembling human $\mathrm{DMD}^{30,31}$. These mice showed an apparently small body size compared with the $m d x$ mice (Supplementary Fig. 5a), with prominent skeletal muscle degeneration that remained to a late stage of post-natal growth (Supplementary Fig. 5b). Total myocyte number in TA muscle was also decreased from 2 to 5 weeks of age in these $m d x / u_{t r n}{ }^{-1}-$ mice, and recovered thereafter (Supplementary Fig. 5c), and central nuclear myocytes a

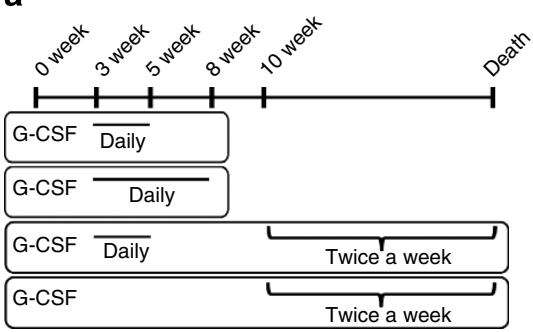

b

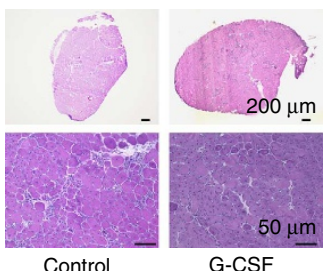

C

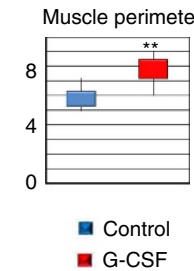

d

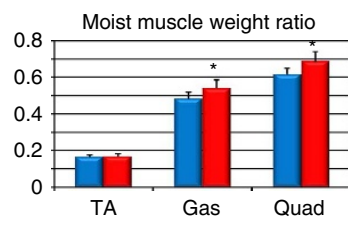

e

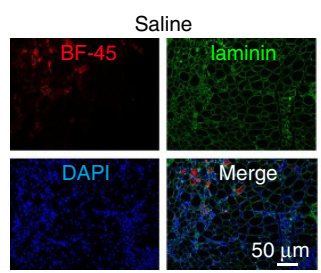

g

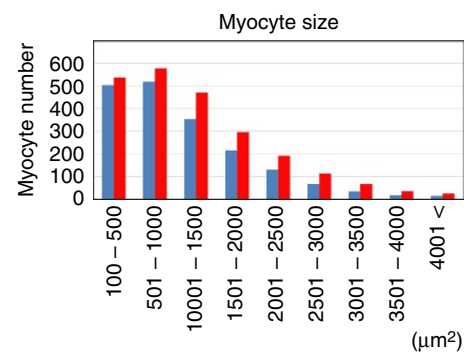

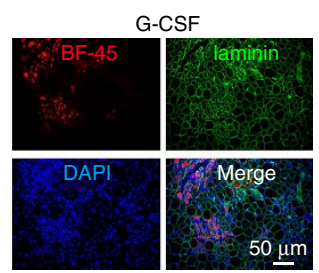

$\mathbf{f}$

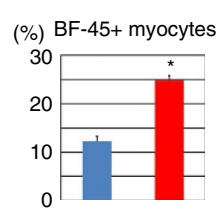

j

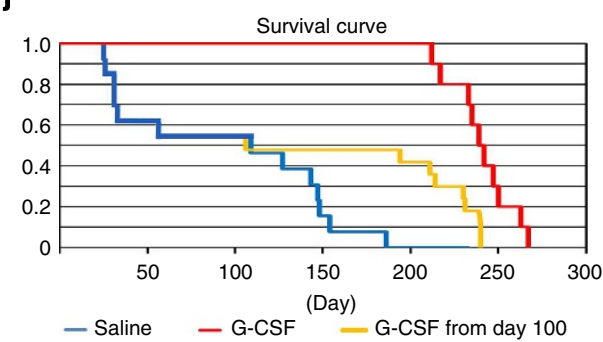

h
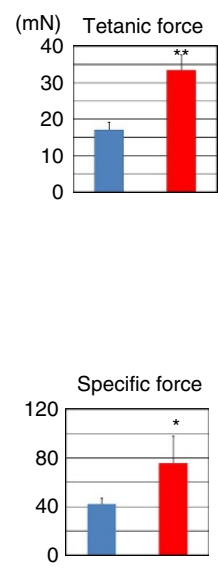

Figure 9 | G-CSF improves the survival of mice with severe muscular dystrophy. (a) Time course of G-CSF administration. (b) Haematoxylin and eosin staining of cross-sections of TA muscle from $m d x / u_{t r n}^{-/-}$mice at 5 weeks of age (left: control; right: G-CSF administration). Scale bar, $200 \mu \mathrm{m}$ (upper panel); $50 \mu \mathrm{m}$ (lower panel). (c) Perimeters of cross-sections of TA muscle at 5 weeks of age. Data are shown as box plot. Top, maximum; bottom, minimum. ${ }^{\star \star} P<0.01$; unequal variance Student's $t$-test; $n=6$ per group. (d) Ratios of moist muscle weight to body weight at 5 weeks of age. Data are shown as mean \pm s.d. ${ }^{*} P<0.05$; equal variance Student's $t$-test; $n=9$ per group. (e) Immunostaining of cross-sections of TA muscle for BF- 45 (red), laminin (green) and DAPI (blue) at 4 weeks of age. Scale bar, $50 \mu \mathrm{m}$. (f) The percentages of BF-45-positive myocytes in cross-sections of TA muscle at 4 weeks of age. Data are shown as mean \pm s.d. ${ }^{\star} P<0.01$; equal variance Student's $t$-test; $n=5$ per group. (g) The individual fibre cross-sectional areas of the myocytes in TA muscle at 5 weeks of age. Data are shown as mean; $n=6$ per group. (h) Tetanic force examination of ex vivo EDL muscle tension at 5 weeks of age is presented. Data are shown as mean \pm s.d. ${ }^{\star \star} P<0.01$; unequal variance Student's $t$-test; $n=5$ per group. (i) Specific force results, represented by tetanic force per muscle size ratios. Data are shown as mean \pm s.d. ${ }^{\star} P<0.05$; unequal variance Student's $t$-test; $n=5$ per group. (j) Kaplan-Meier survival curve of $m d x /$ utrn ${ }^{-/-}$mice without G-CSF $(n=13)$, with G-CSF $(n=10)$ or with G-CSF administration starting at day 100 $(n=9)$. DAPI, 4',6-diamidino-2-phenylindole dihydrochloride. 
were apparent after 3 weeks (Supplementary Fig. 5d). G-CSFR was also expressed in regenerating myocytes in the $m d x / u_{t r n}^{-1-}$ mice (Supplementary Fig. 5e).

To elucidate whether G-CSF improves skeletal muscle regeneration in these mice, we i.p. injected G-CSF using several schedules (Fig. 9a). After daily G-CSF injection from 3 to 5 weeks of age, muscle size was significantly increased in the G-CSFtreated animals at 5 weeks of age (Fig. 9b,c). G-CSF administration also significantly increased the moist muscle weight ratio of the gastrocnemius and quadriceps muscles in $m d x / u_{t r n}{ }^{-1}$ mice (Fig. 9d), as well as the numbers of regenerated myocytes and total myocytes in TA muscle (Supplementary Fig. 6a,b; Fig. 9g). The number of BF- $45+$ myocytes was significantly increased by G-CSF treatment (Fig. 9e,f). The population of PAX7 + cell was also significantly increased by G-CSF treatment (Supplementary Fig. 6c,d). Ex vivo muscle functional analysis showed that tetanic and specific forces were significantly increased by G-CSF administration (Fig. 9h,i). These data suggested that G-CSF improves skeletal muscle regeneration and muscle function in $m d x /$ utrn $^{-1-}$ mice.

The $m d x / u_{t r n}-1-$ mice died at $\sim 3$ to 5 weeks of age and after 100 days of age, resulting in $100 \%$ lethality by 180 days (Fig. 9j, blue line). Finally, we showed that long-term G-CSF treatment could notably improve the survival of $m d x / \mathrm{utrn}^{-1-}$ mice, with G-CSF injected daily from 3 to 5 weeks of age and twice a week after 10 weeks of age (Fig. 9j, red line). It is clinically important to treat developed DMD; hence, the G-CSF was also injected from 10 weeks of age. G-CSF administration prolonged the survival of developed DMD model mice (Fig. 9j, yellow line). Whole-body plethysmography then indicated that G-CSF administration significantly increased respiratory function, measured by tidal volume (TV), in the $m d x /$ trn $^{-1}$ - mice injected daily from 3 to 8 weeks of age (Supplementary Fig. 6e). The G-CSF-treated $m d x /$ utrn $^{-1}$ - mice also showed improved physical activity at 150 days of age (Supplementary Movie). Although cardiomyopathy is one of the fatal phenotypes of DMD, heart morphology is not affected by G-CSF treatment (Supplementary Fig. 6f,g). These data strongly suggested that G-CSF improves skeletal muscle regeneration and function, and supports the survival of DMD model mice.

\section{Discussion}

Research over many years has sought to understand the mechanism of skeletal muscle stem cell behaviour and elucidate the precise pathogenesis underlying DMD and, in turn, to develop effective therapies. Although many promising therapeutic strategies have been developed in animal experiments ${ }^{32}$, most human clinical trials failed to show significant efficacy in patients with $\mathrm{DMD}^{12,14,33-35}$. In addition, with the exception of glucocorticoids, beneficial results with immunosuppressants were inconclusive ${ }^{36-39}$. Indeed, the precise mechanism by which glucocorticoids increase muscle strength in DMD is not known and its clinical effects are generally not sufficient. Improved or additional therapies for DMD are therefore urgently needed. G-CSF is a well-known cytokine that recruits hematopoietic cells ${ }^{40}$. The safety and side effects in humans were widely known from previously established clinical applications, which enable us to use G-CSF early in clinics. Our studies now highlight apparently encouraging the effects of G-CSF on DMD. In this work, we showed that G-CSFR is asymmetrically distributed in some activated SCs, and G-CSF successfully increases the population of SCs at the multi-differentiating stages. We successfully showed that G-CSF administration markedly improves the phenotype of DMD model mice.

The quiescent state in $\mathrm{SC}$ is required for the long-term maintenance of skeletal muscle ${ }^{41}$. We showed that G-CSF increased the PAX7 + /MYOD - self-renewal SCs. There are quiescent SCs among PAX7 + /MYOD - SCs, but it remains unclear whether G-CSF would affect the quiescent SC population. Because G-CSFR is not expressed in PAX7 + /MYOD - SCs, G-CSF signalling would not be directly involved in the transition from activated SCs to PAX7 + /MYOD - SCs. Furthermore, it remains unclear how G-CSF signalling increases the population of PAX7 + /MYOD - SCs. And intrinsic differences among heterogenic satellite stem cells are still unclear and a practical marker to distinguish the subpopulations of satellite stem cells is still missing. Further precise elucidation of molecular mechanisms may directly facilitate control of the quiescent SC pool.

As a study limitation, we could not directly show that bone marrow cells would not be involved in the phenotype of DMD model. To determine whether bone marrow cells have a role in early lethality of haploinsufficiency for G-CSFR in the dystrophic background, we performed bone marrow transplantation using $m d x / c s f 3 r+/-$ mice of $\sim 3-4$ weeks of age. There is no established method of bone marrow transplantation for pups earlier than those ages. We selected active eight $m d x / c s f 3 r+1-$ mice of $\sim 3-4$ weeks of age and successfully performed bone marrow transplantation. One of eight $m d x / c s f 3 r+/-$ mice died at 3 weeks after wild-type bone marrow transplantation by 8 weeks of age, which is similar to the survival rate of active $m d x /$ $c s f 3 r+1-$ mice without bone marrow transplantation. Although wild-type bone marrow cells could not rescue the lethality of $m d x / c s f 3 r+1-$ mice, bone marrow transplantation would preferably be performed in $m d x / c s f 3 r+/-$ mice 0 days of age; alternatively, skeletal muscle-specific G-CSFR-deficient mice would ideally be generated.

We confirmed that the G-CSF/G-CSFR system is critically involved in muscle regeneration during the course of DMD in several mouse models. Given our findings, it is surprising that haploinsufficiency of G-CSFR leads to early lethality in $m d x$ mice, indicating that dose reduction of the G-CSF/G-CSFR system cannot support the balance between degeneration and regeneration in these mouse models. We also showed that G-CSF administration markedly improves the phenotype of $m d x /$ utrn $^{-1-}$ mice. Together, our data suggested that G-CSF could improve the phenotype of human DMD and could consequently form the basis of novel and effective therapies in the near future.

\section{Methods}

Mice. C57BL/6-background $m d x$ mice were provided by $\mathrm{Dr}$ T. Sasaoka (Niigata University Niigata, Japan). The utrophin-knockout mice were a kind gift from Dr Kay E Davies (University of Oxford, Oxford, UK) ${ }^{31}$. The dystrophin-utrophin double-knockout male homozygous (dystrophin ${ }^{-/ Y}(m d x)$, utrophin $\left.^{-1-}\right)$ mice were obtained by crossing those heterozygous $\left(m d x\right.$, utrophin $\left.{ }^{+/-}\right)$mice. The csf $3 r-1-$ mice were kindly gifted by Dr Daniel C. Link (Washington University School of Medicine, St Louis) ${ }^{28}$. Mice deficient in both dystrophin and csf3r were derived by the following breeding program: male csf3r-knockout $(\operatorname{csf} 3 r-/-)$ mice were mated with female $\mathrm{C} 57 \mathrm{~B} / 6 \mathrm{~m} d x\left(\right.$ dystrophin $\left.{ }^{-/ X}\right)$ mice, resulting in all F1-generation mice being heterozygous for $\operatorname{csf} 3 r\left(\operatorname{csf} 3 r^{+/-}\right)$and either heterozygous for the $m d x$ mutation (dystrophin ${ }^{-/ X}$, females) or hemizygous for the $m d x$ mutation (dystrophin ${ }^{-/ Y}$, males). All data were obtained from P1 to 20 weeks of age male mice. All experimental procedures and protocols were approved by the Animal Care and Use Committee of Keio University and conformed to the NIH Guidelines for the Care and Use of Laboratory Animals.

Genotyping. DNA was isolated from mouse tails using the Easy-DNA Kit (Invitrogen, CA, USA). PCR analysis to identify mutant dystrophin alleles used a forward primer complementary to mouse dystrophin (5'-CATAGTTATTAATG CATAGATATTCAG- $\left.3^{\prime}\right)$ and reverse primers complementary to the wild-type allele (5'-GTCACTCAGATAGTTGAAGCCATTTAG-3') or mouse mutant dystrophin allele ( $5^{\prime}$-GTCACTCAGATAGTTGAAGCCATTTAA- $\left.3^{\prime}\right)$. PCR cycling conditions for both the reactions were as follows: $95^{\circ} \mathrm{C}$ for $4 \mathrm{~min} ; 34$ cycles of $95^{\circ} \mathrm{C}$ for $1 \mathrm{~min}, 55^{\circ} \mathrm{C}$ for $1 \mathrm{~min}$ and $72^{\circ} \mathrm{C}$ for $1 \mathrm{~min}$, and $72^{\circ} \mathrm{C}$ for $10 \mathrm{~min}$. Each reaction yielded a 275 -bp product. All PCR reactions were performed using the GoTaq DNA Polymerase kit (Promega, Madison, WI, USA). PCR analysis to assess the 
utrophin-knockout status used a forward primer complementary to exon 7 of mouse utrophin ( $5^{\prime}$-GTGAAGGATGTCATGAAAG- $\left.3^{\prime}\right)$ and reverse primers complementary either to intron 7 (5'-TGAAGTCCGAAAGAGATACC-3') or to the PGK promoter located within the Neo-knockout cassette (5'-ACGAGACTAGT GAGACGTGC- $3^{\prime}$ ). PCR reactions were carried out under the following conditions: 35 cycles of $94^{\circ} \mathrm{C}$ for $30 \mathrm{~s}, 57^{\circ} \mathrm{C}$ for $30 \mathrm{~s}$ and $72^{\circ} \mathrm{C}$ for $25 \mathrm{~s}$. PCR analysis to determine mutant csf3r alleles used a forward primer complementary to mouse csf3r $\left(5^{\prime}\right.$-AGTTCACCAGGCAGGTGAGT- $\left.3^{\prime}\right)$ and reverse primers complementary to wild-type alleles ( $5^{\prime}$-GTAGGCCTAGTTCATACCTG-3') or mouse mutant $c s f 3 r$ alleles $\left(5^{\prime}\right.$-TCCAGACTGCCTTGGGAAAA- $\left.3^{\prime}\right)$. The PCR cycling conditions for both reactions were as follows: $95^{\circ} \mathrm{C}$ for $4 \mathrm{~min}$, followed by 30 cycles of $95^{\circ} \mathrm{C}$ for $30 \mathrm{sec}, 60^{\circ} \mathrm{C}$ for $30 \mathrm{sec}$ and $72{ }^{\circ} \mathrm{C}$ for $45 \mathrm{sec}$. Each reaction yielded a 279-bp product for the wild-type allele and a 366-bp product for the mutant csf3r allele. All PCR reactions were conducted using the Taq DNA Polymerase kit (TaKaRa, Shiga, Japan).

G-CSF treatment. G-CSF (Neutrogin, $100 \mu$; Chugai, Tokyo, Japan) was diluted in $1 \mathrm{ml}$ of saline (concentration of $100 \mu \mathrm{g} \mathrm{ml}^{-1}$ ). Short-term treatment for $\mathrm{mdx}$ mice involved daily i.p. injections of $5 \mu \mathrm{g}$ G-CSF from 3 to 5 weeks of age using a 27-gauge needle. Middle-term treatment for $m d x / \mathrm{utrn}^{-1-}$ mice involved daily i.p. injections from 3 to 8 weeks of age at $5 \mu$ g using a 27-gauge needle. Long-term treatment for $m d x / u t r n^{-1}$ mice involved daily i.p. injections from 3 to 5 weeks of age and twice weekly from 10 weeks of age at $5 \mu$ g using a 27 -gauge needle. Late treatment for $m d x / u_{t r n}{ }^{-1-}$ mice involved twice weekly i.p. injections from 10 weeks of age at $5 \mu \mathrm{g}$ using a 27 -gauge needle.

Histological analysis. Frozen section of TA muscle was stained by haematoxylin and eosin to measure the ratio of muscle regeneration. The fibre cross-sectional area was measured using software (BZ-H1C: Keyence, Osaka, Japan) from lamininstained cross-sections of TA muscle. Micrographs were taken from each section at, $\times 10$ and $\times 20$ magnification with a digital camera (BIOREVO: Keyence). Evans blue (Sigma-Aldrich, MO, USA) was dissolved in PBS $(0.15 \mathrm{M} \mathrm{NaCl}, 10 \mathrm{mM}$ phosphate buffer, $\mathrm{pH} 7.0$ ). Mice were i.p. injected with $0.5 \mathrm{ml} 0.1 \%$ Evans blue at P1 using a 27 -gauge needle and were killed $3 \mathrm{~h}$ later.

Immunofluorescence. All immunofluorescence was carried out on 6- $\mu$ m-thick frozen sections, myofibres and SCs. The frozen sections were fixed with acetone (WAKO, Osaka, Japan) for $20 \mathrm{~min}$ at $-30^{\circ} \mathrm{C}$. Myofibres and SCs were fixed with $4 \%$ paraformaldehyde for $20 \mathrm{~min}$ at room temperature. All samples were incubated with $0.1 \%$ Triton X-100 for $5 \mathrm{~min}$ at room temperature, washed and then incubated with the following primary antibodies: anti-G-CSF receptor (sc-9173, 1:50: Santa Cruz Biotechnology, CA, USA; sc-323898, 1:100, Santa Cruz Biotechnology), antilaminin 2 (L0663, 1:1000, Sigma-Aldrich), anti-laminin (L9393, 1:1000, SigmaAldrich), anti-BF-45 (1:30, Developmental Studies Hybridoma Bank, IA, USA), anti-MyoD (sc-32758, 1:200, mouse; sc-304, 1:200, Santa Cruz Biotechnology), anti-Pax7 (sc-81648, 1:100, 1:40, Santa Cruz Biotechnology), anti-myogenin (ab1835, 1:100, Abcam, Camb, UK; sc-576 1:100, Santa Cruz Biotechnology), anti-syndecan-4 (ab24511, 1:100, Abcam), anti-phospho-SAPK/JNK (\#9251 1:100, rabbit, Cell Signaling Technology, MA, USA) and anti-phospho-p38 (sc-7973, 1:100, mouse, Santa Cruz Biotechnology). After overnight incubation, bound antibodies were visualized with a secondary antibody conjugated with Alexa 488, Alexa 546 or Alexa 633 (Life Technologies, CA, USA). Nuclei were stained with 4',6-diamidino-2-phenylindole dihydrochloride (Life technologies). For Pax7 staining, an M.O.M. kit (Vector Laboratories) was used to block the endogenous mouse IgG. The images were recorded using a confocal laser microscope system (Carl Zeiss, Jena, Germany) and BIOREVO (Keyence).

Muscle force measurements. EDL muscles were isolated and removed from 5 -week-old mice. The muscles were carefully mounted in a chamber filled with PBS $\left(95 \% \mathrm{O}_{2}\right.$ and $5 \% \mathrm{CO}_{2}$ ) and maintained at $30^{\circ} \mathrm{C}$. One tendon of the muscle was attached to a steel hook in the chamber, and the other was tied to the lever arm of a dual-mode servomotor system (Electronic Stimulator; NIHON KOHDEN, Tokyo, Japan) via 5-0 surgical silk. The muscles were stretched to the length at which a single twitch and tetanic force showed the highest amplitude (optimal length; $L_{\mathrm{o}}$ ). The corresponding tetanic force was then measured at $150 \mathrm{~Hz}$ over 5 -s intervals for a total of $20 \mathrm{~min}$, with a rest period of $120 \mathrm{~s}$ to change the buffer. The muscles were adjusted to the optimum length $\left(L_{\mathrm{o}}\right)$ before all force measurements. For comparative purposes, all force measurements were expressed per the total muscle unit cross-sectional area, which was calculated by dividing the muscle mass by the product of the length and the mammalian skeletal muscle density $\left(1.056 \mathrm{mg} \mathrm{mm}^{-3}\right)$. The specific force $\left(\mathrm{N} \mathrm{cm}^{-2}\right)$ was calculated with the muscle density assumed as $1.056 \mathrm{~g} \mathrm{~cm}^{-3}$.

Exercise tolerance tests. Mice were subjected to an exhaustion treadmill test by placing them on the belt of a one-lane motorized treadmill (MK-680S Treadmill for Rats \& Mice; Muromachi Kikai, Tokyo, Japan). The test was started at an incline of 0 at $5 \mathrm{~m} \mathrm{~min}^{-1}$ for $5 \mathrm{~min}$; thereafter, the speed was increased by $1 \mathrm{~m} \mathrm{~min}^{-1}$ every minute. The end point of the test was when the mouse remained on the shocker plate for $>20 \mathrm{~s}$.

Whole-body plethysmography. Animals were placed in a free-moving chamber that was connected to a high-gain differential pressure transducer (Valydine MP45, Validyne, North Ridge, CA, USA). As the animal breathed, changes in the pressure were converted to signals representing the TV; these signals were amplified (BMA 830; CWE, Ardmore, PA, USA), recorded on a strip-chart recorder (Dash 10; Astro-Med, West Warwick, RI, USA) and stored in a computer with respiratory acquisition software for analysis. $\mathrm{O}_{2}$ consumption and $\mathrm{CO}_{2}$ production were measured by the open-circuit method using Beckman OM-14 and LB-2 analysers. The parameter TV was analysed in real time, then average values were calculated five times every $2 \mathrm{~min}$ for each serial 3 -h period. The TV was normalized by dividing by the body weight.

Myofibre isolation and culture. Single myofibres were isolated from EDL muscles and digested in DMEM GltaMax (Gibco, CA, USA) with $0.2 \%$ type 1 collagenase at $37^{\circ} \mathrm{C}$ for $90 \mathrm{~min}$. Fibres were liberated by trituration in DMEM medium with Pasteur pipettes. Isolated myofibres were cultured in suspension in serum-coated dishes. The fibre medium contained $20 \%$ foetal bovine serum (Sigma-Aldrich), $1 \%$ chick embryo extract (US Biological, MA, USA) $1 \%$ penicillin-streptomycin (Gibco), and DMEM GltaMax (Gibco). Single myofibres were incubated at $37^{\circ} \mathrm{C}$ with $5 \% \mathrm{CO}_{2}$ for the indicated times. For the treatments, G-CSF (Neutrogin; Chugai) was added to the fibre medium to a concentration of $0.375 \mathrm{ng} \mathrm{ml}^{-1}, \mathrm{p} 38$ inhibitor (SB203580, CST) was added to the fibre medium at a concentration of $10 \mu \mathrm{M}$ and JNK inhibitor (SP600125, Cell Signaling Technology) was added to the fibre medium at a concentration of $5 \mu \mathrm{M}$.

Isolation of SCs. We obtained SCs using single myofibre culture, during which the SCs began to migrate on Matrigel (BD Biosciences, CA, USA)-coated dishes. Single SCs were then placed in Matrigel-coated glass-base dishes (Asahi Glass, Tokyo, Japan) and were maintained in fibre medium at $37^{\circ} \mathrm{C}$ with $5 \% \mathrm{CO}_{2}$ for 3 days to allow SCs to migrate off the fibre and onto the Matrigel-coated dishes.

Statistical analysis. Values are reported as the means \pm s.d. The data were analysed using StatView J-4.5 software. Comparisons between the two groups were performed with Student's $t$-test. Comparisons among the groups were performed by one-way analysis of variance with Bonferroni's post hoc test. Scheffe's $F$-test was used to determine the level of significance. The probability level accepted for significance was ${ }^{\star} P<0.05,{ }^{*} P<0.01$.

\section{References}

1. Rocheteau, P., Gayraud-Morel, B., Siegl-Cachedenier, I., Blasco, M. A. \& Tajbakhsh, S. A Subpopulation of adult skeletal muscle stem cells retains all template DNA strands after cell division. Cell 148, 112-125 (2012).

2. Troy, A. et al. Coordination of satellite cell activation and self-renewal by Par-complex-dependent asymmetric activation of $\mathrm{p} 38 \alpha / \beta$ MAPK. Cell Stem Cell 11, 541-553 (2012).

3. Kawabe, Y.-i., Wang, Y. u. X., McKinnell, I. W., Bedford, M. T. \& Rudnicki, M. A. Carm1 regulates Pax7 transcriptional activity through MLL1/2 recruitment during asymmetric satellite stem cell divisions. Cell Stem Cell 11, 333-345 (2012).

4. Cheung, T. H. et al. Maintenance of muscle stem-cell quiescence by microRNA-489. Nature 482, 524-528 (2012).

5. Relaix, F. \& Zammit, P. S. Satellite cells are essential for skeletal muscle regeneration: the cell on the edge returns centre stage. Development 139, 2845-2856 (2012).

6. Yuasa, S. et al. Transient inhibition of BMP signaling by Noggin induces cardiomyocyte differentiation of mouse embryonic stem cells. Nat. Biotechnol. 23, 607-611 (2005).

7. Yuasa, S. et al. Zac1 is an essential transcription factor for cardiac morphogenesis. Circ. Res. 106, 1083-1091 (2010).

8. Shimoji, K. et al. G-CSF promotes the proliferation of developing cardiomyocytes in vivo and in derivation from ESCs and iPSCs. Cell Stem Cell 6, 227-237 (2010).

9. Hara, M. et al. G-CSF influences mouse skeletal muscle development and regeneration by stimulating myoblast proliferation. J. Exp. Med. 208, 715-727 (2011).

10. Stratos, I., Rotter, R., Eipel, C., Mittlmeier, T. \& Vollmar, B. Granulocyte-colony stimulating factor enhances muscle proliferation and strength following skeletal muscle injury in rats. J. Appl. Physiol. 103, 1857-1863 (2007).

11. Emery, A. E. H. The muscular dystrophies. Lancet 359, 687-695 (2002).

12. Bushby, K. et al. Diagnosis and management of Duchenne muscular dystrophy, part 1: diagnosis, and pharmacological and psychosocial management. Lancet Neurol. 9, 77-93 (2010).

13. Emery, A. E. H. Population frequencies of inherited neuromuscular diseases--a world survey. Neuromuscul. Disord. 1, 19-29 (1991). 
14. Muntoni, F., Torelli, S. \& Ferlini, A. Dystrophin and mutations: one gene, several proteins, multiple phenotypes. Lancet Neurol. 2, 731-740 (2003).

15. Hoffman, E. P. et al. Characterization of dystrophin in muscle-biopsy specimens from patients with Duchenne's or Becker's muscular dystrophy. N. Engl. J. Med. 318, 1363-1368 (1988).

16. Petrof, B. J., Shrager, J. B., Stedman, H. H., Kelly, A. M. \& Sweeney, H. L. Dystrophin protects the sarcolemma from stresses developed during muscle contraction. Proc. Natl Acad. Sci. USA 90, 3710-3714 (1993).

17. Shi, X. \& Garry, D. J. Muscle stem cells in development, regeneration, and disease. Genes Dev. 20, 1692-1708 (2006).

18. Olguin, H. C., Yang, Z., Tapscott, S. J. \& Olwin, B. B. Reciprocal inhibition between Pax7 and muscle regulatory factors modulates myogenic cell fate determination. J. Cell Biol. 177, 769-779 (2007).

19. Bentzinger, C. F. et al. Fibronectin regulates Wnt7a Signaling and satellite cell expansion. Cell Stem Cell 12, 75-87 (2013).

20. Tanaka, K. K. et al. Syndecan-4-expressing muscle progenitor cells in the SP engraft as satellite cells during muscle regeneration. Cell Stem Cell 4, 217-225 (2009).

21. Zammit, P. S. et al. Muscle satellite cells adopt divergent fates: a mechanism for self-renewal? J. Cell Biol. 166, 347-357 (2004).

22. Yablonka-Reuveni, Z., Day, K., Vine, A. \& Shefer, G. Defining the transcriptional signature of skeletal muscle stem cells. J. Anim. Sci. 86, E207-E216 (2008)

23. Siegel, A., Kuhlmann, P. \& Cornelison, D. Muscle satellite cell proliferation and association: new insights from myofiber time-lapse imaging. Skelet. Muscle 1, 7 (2011).

24. Jones, N. C. et al. The $\mathrm{p} 38 \alpha / \beta$ MAPK functions as a molecular switch to activate the quiescent satellite cell. J. Cell Biol. 169, 105-116 (2005).

25. Alter, J., Rozentzweig, D. \& Bengal, E. Inhibition of myoblast differentiation by tumor necrosis factor $\alpha$ is mediated by c-Jun $\mathrm{N}$-terminal kinase 1 and leukemia inhibitory factor. J. Biol. Chem. 283, 23224-23234 (2008).

26. Evans, N. P., Misyak, S. A., Robertson, J. L., Bassaganya-Riera, J. \& Grange, R. W. Dysregulated intracellular signaling and inflammatory gene expression during initial disease onset in Duchenne muscular dystrophy. Am. J. Phys. Med. Rehabil. 88, 502-522 (2009).

27. Schiaffino, S. et al. Embryonic and neonatal myosin heavy chain in denervated and paralyzed rat skeletal muscle. Dev. Biol. 127, 1-11 (1988).

28. Richards, M. K., Liu, F., Iwasaki, H., Akashi, K. \& Link, D. C. Pivotal role of granulocyte colony-stimulating factor in the development of progenitors in the common myeloid pathway. Blood 102, 3562-3568 (2003).

29. Hermans, M. H. A. et al. Perturbed granulopoiesis in mice with a targeted mutation in the granulocyte colony-stimulating factor receptor gene associated with severe chronic neutropenia. Blood 92, 32-39 (1998).

30. Grady, R. M. et al. Skeletal and cardiac myopathies in mice lacking utrophin and dystrophin: a model for Duchenne muscular dystrophy. Cell 90, 729-738 (1997).

31. Deconinck, A. E. et al. Utrophin-dystrophin-deficient mice as a model for Duchenne muscular dystrophy. Cell 90, 717-727 (1997).

32. Muntoni, F. \& Wells, D. Genetic treatments in muscular dystrophies. Curr. Opin. Neurol. 20, 590-594 (2007).

33. Bushby, K., Muntoni, F., Urtizberea, A., Hughes, R. \& Griggs, R. Report on the 124th ENMC International Workshop. Treatment of Duchenne muscular dystrophy; defining the gold standards of management in the use of corticosteroids 2-4 April 2004, Naarden, The Netherlands. Neuromuscul. Disord. 14, 526-534 (2004)

34. Drachman, D. B., Toyka, K. V. \& Myer, E. Prednisone in Duchenne muscular dystrophy. Lancet 304, 1409-1412 (1974).

35. Fairclough, R., Bareja, A. \& Davies, K. Progress in therapy for Duchenne muscular dystrophy. Exp. Physiol. 96, 1101-1113 (2011).

36. Ameen, V. \& Robson, L. G. Experimental models of duchenne muscular dystrophy: relationship with cardiovascular disease. Open Cardiovasc. Med. J. 4, 265-277 (2010).

37. Quinlan, J. G. et al. Evolution of the mdx mouse cardiomyopathy: physiological and morphological findings. Neuromuscul. Disord. 14, 491-496 (2004).

38. Griggs, R. C. Duchenne muscular dystrophy: an important negative trial. Lancet Neurol. 9, 1038-1039 (2010).

39. Sharma, K. R., Mynhier, M. A. \& Miller, R. G. Cyclosporine increases muscular force generation in Duchenne muscular dystrophy. Neurology 43, 527-532 (1993).

40. Cottler-Fox, M. H. et al. Stem cell mobilization. Hematology Am. Soc. Hematol. Educ. Program 1, 419-437 (2003).

41. Wang, Y. X., Dumont, N. A. \& Rudnicki, M. A. Muscle stem cells at a glance. J. Cell. Sci. 127, 4543-4548 (2014).

\section{Acknowledgements}

This study was supported in part by research grants from The Ministry of Education, Science and Culture, Japan; The Program for Promotion of Fundamental Studies in Health Science of the National Institute of Biomedical Innovation; The Ministry of Health Labour and Welfare; Health Labour Sciences Research Grant; Nakatomi Foundation; Japan Heart Foundation/Novartis Grant for Research Award on Molecular and Cellular Cardiology; SENSHIN Medical Research Foundation; Kimura Memorial Heart Foundation Research Grant; Japan Intractable Diseases Research Foundation, Japan; The Cell Science Research Foundation; The Tokyo Biochemical Research Foundation; Suzuken Memorial Foundation; and The Japan Foundation for Applied Enzymology.

\section{Author contributions}

S.Y. designed the research, N.H., S.Y., Y.S., M.H., N.I., H.H., D.K., T.S., S.T., M.K., A.K., S.K., M.T., Y.S., S.O., T.E., J.E. and T.S. performed the research, N.H., S.Y., Y.S., S.T. and K.F. analysed the data and S.Y. wrote the paper.

\section{Additional information}

Supplementary Information accompanies this paper at http://www.nature.com/ naturecommunications

Competing financial interests: The authors declare no competing financial interests.

Reprints and permission information is available online at http://npg.nature.com/ reprintsandpermissions/

How to cite this article: Hayashiji, N. et al. G-CSF supports long-term muscle regeneration in mouse models of muscular dystrophy. Nat. Commun. 6:6745 doi: 10.1038/ncomms7745 (2015). 


\section{Erratum: G-CSF supports long-term muscle regeneration in mouse models of muscular dystrophy}

Nozomi Hayashiji, Shinsuke Yuasa, Yuko Miyagoe-Suzuki, Mie Hara, Naoki Ito, Hisayuki Hashimoto,

Dai Kusumoto, Tomohisa Seki, Shugo Tohyama, Masaki Kodaira, Akira Kunitomi, Shin Kashimura, Makoto Takei, Yuki Saito, Shinichiro Okata, Toru Egashira, Jin Endo, Toshikuni Sasaoka, Shin'ichi Takeda \& Keiichi Fukuda

Nature Communications 6:6745 doi: 10.1038/ncomms7745 (2015); Published 13 April 2015; Updated 18 Jun 2015.

The immunofluorescence images in Fig. 1b of the PDF version of this Article were inadvertently duplicated from Fig. 1a during the production process. The correct version of Fig. 1 appears below; the HTML version of the paper was correct from the time of publication. 
a
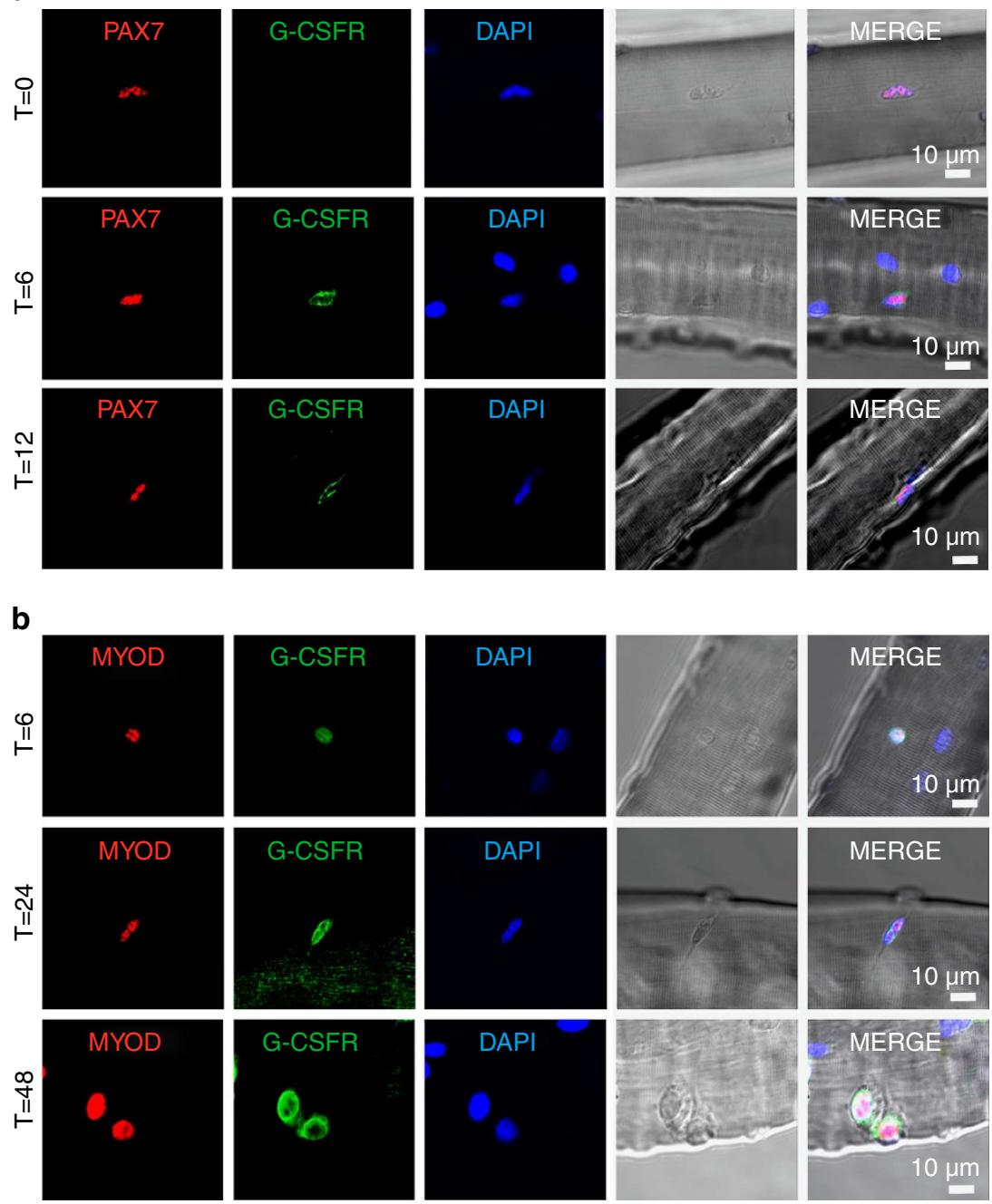

C

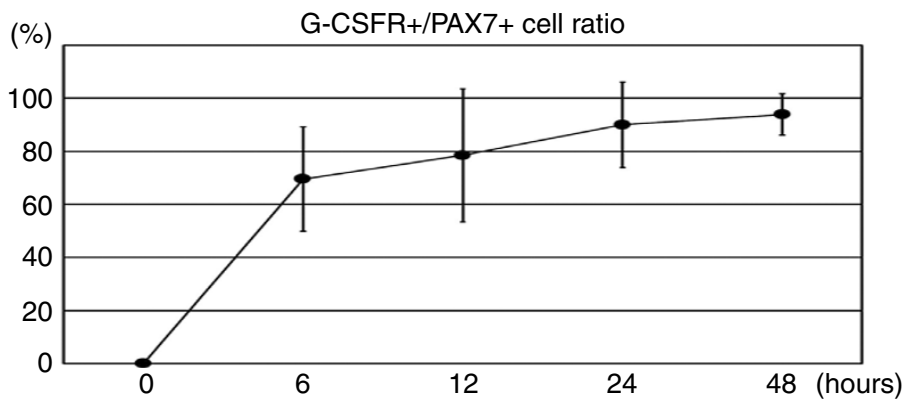

Figure 1 